\title{
Interplay of gas and ice during cloud evolution
}

\author{
S. Hocuk ${ }^{1,2}$ and S. Cazaux ${ }^{1}$ \\ 1 Kapteyn Astronomical Institute, University of Groningen, PO Box 800, 9700 AV Groningen, The Netherlands \\ e-mail: cazaux@astro.rug.nl \\ 2 Max-Planck-Institüt für extraterrestrische Physik, Giessenbachstrasse 1, 85748 Garching, Germany \\ e-mail: seyit@mpe.mpg.de
}

Received 30 June 2014 / Accepted 9 January 2015

\begin{abstract}
During the evolution of diffuse clouds to molecular clouds, gas-phase molecules freeze out on surfaces of small dust particles to form ices. On dust surfaces, water is the main constituent of the icy mantle in which a complex chemistry is taking place. We aim to study the formation pathways and the composition of the ices throughout the evolution of diffuse clouds. For this purpose, we used time-dependent rate equations to calculate the molecular abundances in the gas phase and on solid surfaces (onto dust grains). We fully considered the gas-dust interplay by including the details of freeze-out, chemical and thermal desorption, and the most important photo-processes on grain surfaces. The difference in binding energies of chemical species on bare and icy surfaces was also incorporated into our equations. Using the numerical code FLASH, we performed a hydrodynamical simulation of a gravitationally bound diffuse cloud and followed its contraction. We find that while the dust grains are still bare, water formation is enhanced by grain surface chemistry that is subsequently released into the gas phase, enriching the molecular medium. The $\mathrm{CO}$ molecules, on the other hand, tend to gradually freeze out on bare grains. This causes CO to be well mixed and strongly present within the first ice layer. Once one monolayer of water ice has formed, the binding energy of the grain surface changes significantly, and an immediate and strong depletion of gas-phase water and $\mathrm{CO}$ molecules occurs. While hydrogenation converts solid $\mathrm{CO}$ into formaldehyde $\left(\mathrm{H}_{2} \mathrm{CO}\right)$ and methanol $\left(\mathrm{CH}_{3} \mathrm{OH}\right)$, water ice becomes the main constituent of the icy grains. Inside molecular clumps formaldehyde is more abundant than water and methanol in the gas phase, owing its presence in part to chemical desorption.
\end{abstract}

Key words. astrochemistry - hydrodynamics - methods: numerical - stars: formation - dust, extinction - ISM: clouds

\section{Introduction}

Dust grains play a vital role in the making and breaking of molecules in interstellar gas clouds because many chemical reactions proceed much faster on solid surfaces than reactions in the gas phase. As a result, dust can enhance molecular abundances in interstellar gas clouds by acting as a catalyst for the formation of complex molecules (e.g., recent studies by Meijerink et al. 2012; Le Bourlot et al. 2012; Gavilan et al. 2012). However, dust grains can also lock up gas-phase molecules through freeze-out in cold environments (e.g., Jones \& Williams 1984; Lippok et al. 2013). This duality creates an intricate relationship between gas and dust particles. Their involvement in chemical reactions affects the thermodynamic properties of molecular clouds and from this their whole evolution (Goldsmith 2001; Cazaux et al. 2010). The kinetic temperature of a gas cloud is especially sensitive to changes in abundances of the dominant coolants, like CO (Hocuk et al. 2014). These findings highlight the importance of considering the formation of ices and depletion of gas-phase species in models and theories of cloud evolution and star formation.

Ices form on dust grains in the interstellar medium (ISM) during the evolution of interstellar clouds, the progenitors of star-forming regions. Diffuse clouds, where the dust-gas coupling and grain surface chemistry is still negligible, will evolve and undergo phase transitions to form molecular clouds, where freeze-out is effective, and eventually form dense clumps where dust-gas coupling becomes dominant. These are the critical phases that clouds undergo before a star finally forms and which, ultimately, determine the stellar masses at birth. During the evolutionary stages, gas-phase molecules are deposited on grain surfaces to form icy layers, thereby depleting the gaseous molecules. Eventually, the ices will grow thick mantels on dust grains and will remain there until the medium becomes warmer, allowing the species to evaporate back into the gas phase. Heating can be caused by radiation (UV photons, X-rays, or cosmic rays) penetrating the cloud and interacting with the gas, shock-waves injecting kinetic heating, or gravitational collapse where compressional heating ensues and radiation becomes trapped. Hot cores already revealed a glimpse of the rich organic chemistry locked up in the ice mantles (Cazaux et al. 2003), which suddenly became visible as a result of evaporation into the gas phase by protostellar heating.

Depletion of molecular species, such as CO, are also observed toward the formation of prestellar cores (Tafalla et al. 2006; Liu et al. 2013), with depletion factors of up to 80 in dense clumps (Fontani et al. 2012), indicating the presence of thick ice mantles. Observations of cold prestellar cores reported the lack of gas-phase $\mathrm{H}_{2} \mathrm{O}$, demonstrating a much more serious freezeout than previously predicted (van Dishoeck et al. 2011). The presence of frozen water was corroborated by the detection of strong water emission from shocks in protostellar environments (van Dishoeck et al. 2011; Suutarinen et al. 2014). Recent observations of starburst galaxy M82 showed that $\mathrm{CO}_{2}$ and $\mathrm{H}_{2} \mathrm{O}$ ices are present for different physical conditions (Yamagishi et al. 2013). This is intriguing because it is demanding for models to predict high abundances of $\mathrm{CO}_{2}$. The pathway of forming $\mathrm{CO}_{2}$ is still very uncertain (Ioppolo et al. 2013), but the gas-phase route is widely accepted as inefficient. This leads to the notion that $\mathrm{CO}$ ice is not passive. 
Observations of deeply embedded protostars show an anticorrelation between the abundance of CO (depleted) and methanol in the gas (Kristensen et al. 2010). This demonstrates that $\mathrm{CO}$ converts into methanol on the surface of dust grains and is subsequently released into the gas phase. The observed orthoto-para ratio of water in the Orion Bar is also found to be inconsistent with only gas-phase water formation (Choi et al. 2013), indicating that nonthermal processes must be at work on dust grains. An important mechanism to supply the gas phase with ice species is the nonthermal process of photodesorption from either UV or cosmic-ray-induced UV photons. Photons with energies of mostly around $8 \mathrm{eV}$ can directly desorb $\mathrm{CO}$ molecules into the gas phase (Fayolle et al. 2011). Photodesorption is considered as a possible important gas-phase supplier of CO (Muñoz Caro et al. 2010), methanol and formaldehyde (Yusef-Zadeh et al. 2013; Guzmán et al. 2013), water (Keto et al. 2014), $\mathrm{CO}_{2}$ (Öberg et al. 2009; Bahr \& Baragiola 2012), or $\mathrm{O}_{2}$ (Zhen \& Linnartz 2014). Observations by Yildiz et al. (2013) suggest, however, that photodesorption rates need to be increased by a factor of 2 to explain their abundances of $\mathrm{O}_{2}$.

Recent experimental studies have unveiled another nonthermal mechanism, called chemical desorption, that directly converts species formed on dust surfaces into gas-phase species (Garrod et al. 2007; Dulieu et al. 2013). This mechanism occurs for exothermic reactions, where the products cannot thermalize with the dust surface. These findings show that the formation of species through surface reactions does not just lock up species from the gas phase, but will also directly enrich the gas-phase medium and is therefore an integral part of interstellar cloud evolution.

In this work we track the formation of ices during the evolution of an interstellar gas cloud, starting from a diffuse, fully atomic stage until the formation of dense clumps. We observe the formation of the first ice layers on the surfaces of dust grains, report their compositions, and determine the distribution of ices around a dense clump as well as their formation rates. For this purpose, we developed a chemical network using rate equations that incorporates grain surface reactions on two different substrates, bare grain (no ices) surface, and water ice surface. We also consider the processes of chemical and photodesorption (Cazaux et al. 2010; Noble et al. 2012a; Dulieu et al. 2013) and the most recent reactions through quantum tunneling (Oba et al. 2012; Minissale et al. 2013b). The paper is organized as follows: In Sect. 2 we present the code that is used in this work and describe our initial conditions. In Sect. 3 we explain the chemical processes on dust surfaces, describe each process separately, and present our equations. In Sect. 4 we outline the important thermal processes and report the heating and cooling terms used in our calculations. In Sect. 5 we present our results on the composition of the ice layers, show the dominant species formation rates, and give the distribution of ices around a dense clump. We also discuss the implications of our results. In Sect. 6 we conclude and discuss the caveats.

\section{Numerical method}

\subsection{Numerical code}

We performed the numerical simulations with the adaptive-mesh hydrodynamical code FLASH, version 4.0 (Fryxell et al. 2000; Dubey et al. 2009). Our work encompasses a broad range of physics, such as hydrodynamics, chemistry, thermodynamics (using time-dependent heating and cooling rates), turbulence, multispecies, gravity, and radiative transfer for UV. To solve

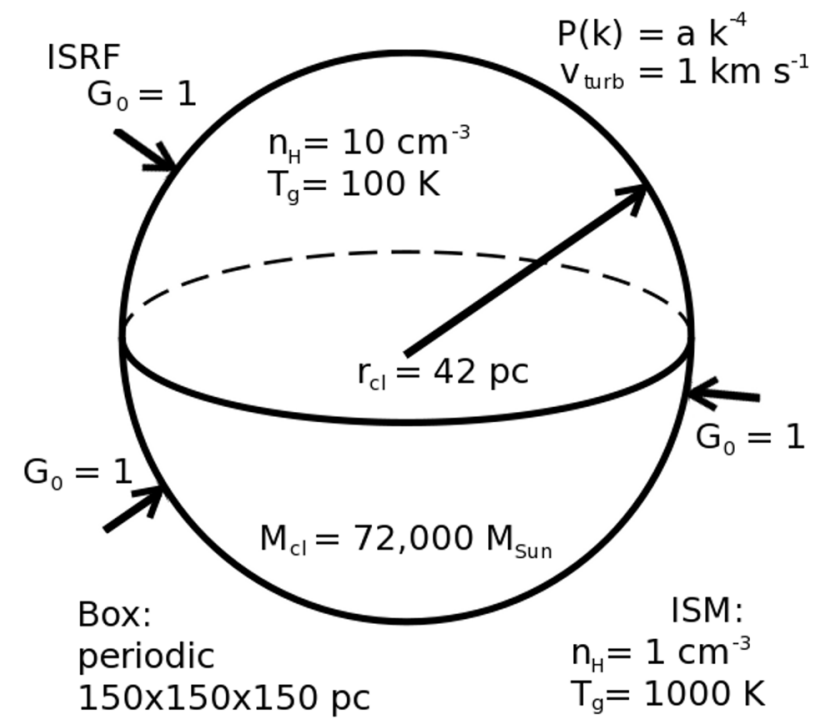

Fig. 1. Initial setup of the diffuse interstellar cloud.

the hydrodynamic equations, we applied the directionally split piecewise-parabolic method (PPM; Colella \& Woodward 1984), which is well-suited to handling the type of calculations in this study. Our research captures the physics that act on small and large scales. These are the micro-sized scales required for the chemical reactions in the gas phase and on grain surfaces and the parsec-sized scales that are necessary to describe star formation processes.

To track multiple fluids, we employed the multispecies unit that is able to follow each species with its own properties. We applied the consistent multifluid advection scheme (Plewa \& Müller 1999) to prevent overshoots in the mass fractions as a result of the PPM advection. The Poisson equations were solved with the Multigrid solver in which gravity is coupled to the Euler equations through the momentum and energy equations. The physics modules are well-tested and were either provided by FLASH or can be found in earlier works, for example, Hocuk \& Spaans $(2010,2011)$ and Hocuk et al. (2014). A comprehensive chemistry and a thermodynamics module were created specifically for this work; they are explained in more detail in Sects. 3 and 4 .

\subsection{Initial conditions}

We created a gravitationally bound diffuse gas cloud in which all hydrogen is in atomic form. Our model cloud starts with a uniform number density of $n_{\mathrm{H}}=10 \mathrm{~cm}^{-3}$ and an initial temperature of $100 \mathrm{~K} . n_{\mathrm{H}}$ is defined as the total hydrogen nuclei number density, that is, $n_{\mathrm{H}}=\rho / m_{\mathrm{H}}$. The interstellar environment including the cloud surface has a temperature of $1000 \mathrm{~K}$ and a number density of $n_{\mathrm{H}}=1 \mathrm{~cm}^{-3}$. We allowed for a smooth, hyperbolic transition for the values from the cloud edge to the surrounding ISM. To follow its chemistry and temperature during its evolution, we placed our model cloud in a 3D cubic box of size $150 \mathrm{pc}^{3}$. We applied periodic boundary conditions to our simulation domain. The spherical cloud has a radius of $42 \mathrm{pc}$ and a total mass of $7.2 \times 10^{4} M_{\odot}$. A graphical display of the initial conditions is given in Fig. 1. 
The diffuse cloud was initiated with turbulent conditions that are representative of the ISM in the Milky Way, $\sigma_{\text {turb }}=$ $1 \mathrm{~km} \mathrm{~s}^{-1}$, with a power spectrum of $P(k) \propto k^{-4}$ following the empirical laws for compressible fluids (Larson 1981; Myers \& Gammie 1999; Heyer \& Brunt 2004). This scaling is also known as Burgers turbulence (Burgers 1939; Bec \& Khanin 2007). The turbulence is decaying and not driven. The cloud is also not supported by turbulence and will contract. The simulated cloud was placed in an environment with a background UV radiation flux of $G_{0}=1$ in terms of the Habing field (Habing 1968). This agrees with the ISM conditions of our Milky Way. The cloud center enjoys column densities of over $10^{21} \mathrm{~cm}^{-3}$, which is equivalent to a visual extinction of $A_{V} \sim 0.5$. We do not consider magnetic fields in this work.

We refined the computational grid that encloses the model cloud to a uniform resolution of $128^{3}$ cells. This yields a spatial resolution of $1.17 \mathrm{pc}$, which corresponds to a Jeans resolution of 44 grid cells per Jeans length for the initial state. The Jeans length is calculated as

$\lambda_{\mathrm{J}}=\left(\frac{\pi \mathrm{c}_{\mathrm{s}}^{2}}{\mathrm{G} \rho}\right)^{\frac{1}{2}} \mathrm{~cm}$.

Here $c_{\mathrm{S}}$ is the sound speed, which, for an ideal gas, can be formulated as $c_{\mathrm{s}}^{2}=\gamma P / \rho=\gamma N_{\mathrm{A}} k_{\mathrm{B}} T / \mu$. In this, $N_{\mathrm{A}}$ and $k_{\mathrm{B}}$ are the Avogadro number and the Boltzmann constant, $\mu$ is the mean molecular mass, which is unity in the initial case, and the parameter $\gamma$ depends on the equation of state (EOS). For a polytropic EOS, the pressure scales as $P \propto \rho^{\gamma}$ (Spaans \& Silk 2000). The polytropic index $\gamma$ will be affected by the thermal balance, that is, heating and cooling, of the cloud and therefore is not a fixed quantity. The sound speed of the diffuse cloud if it is isothermal $(\gamma=1)$ is $c_{\mathrm{s}}=0.91 \mathrm{~km} \mathrm{~s}^{-1}$.

We did not increase grid resolution during the simulation nor allowed for star formation to occur. The spatial resolution will therefore not be as high as contemporary advanced numerical studies of star formation, but our goal is not to resolve the fine details of cloud fragmentation, turbulence, or the direct spatial conditions prior to star formation. Our focus lies on describing the chemical composition (gas + dust) and thermal balance of an evolving gas cloud.

Because the formation of ices can take more than $10^{4}$ years (Cuppen \& Herbst 2007) and is dependent on the changing conditions within the cloud, an equilibrium solution is not acceptable. Within our time-dependent solver, we also did not allow for variations in species densities of over $5 \%$ per iteration. To this end, we employed a very high time resolution with adaptive time-stepping that can extend to a time resolution of three days $(0.01 \mathrm{yr})$ for a simulation that lasts over ten million years. In the most unfavorable case, it is necessary to iterate the chemistry routine more than 10000 times within a hydrodynamical time step. The adaptive chemical time-stepping is handled within a subcycling loop of the hydrodynamical time step to avoid any unnecessary speed loss for the other routines. We let the cloud evolve until it reached $125 \%$ of its theoretical free-fall time, where $t_{\mathrm{ff}}=\sqrt{3 \pi / 32 \mathrm{G} \rho}$. Given the initial conditions of the model cloud, this adds up to a final simulation time of $2.0 \times 10^{7} \mathrm{yr}$.

\section{Analytical method: time-dependent chemistry}

We performed time-dependent rate equations at each grid cell that include both gas and grain surface reactions to compute the chemical composition of the cloud. Our chemical model comprises 42 different species. Of these, 26 are in the gas phase
Table 1. Species and their initial abundances $\left(n_{x_{i}} / n_{\mathrm{H}}\right)$.

\begin{tabular}{|c|c|c|c|}
\hline Species & Initial abundance & Species & Initial abundance \\
\hline $\mathrm{H}$ & 1.0 & $\mathrm{HCO}^{+}$ & 0 \\
\hline $\mathrm{H}^{-}$ & 0 & $\mathrm{H}_{2} \mathrm{CO}$ & 0 \\
\hline $\mathrm{H}^{+}$ & 0 & $\mathrm{CH}_{3} \mathrm{O}$ & 0 \\
\hline $\mathrm{H}_{2}$ & 0 & $\mathrm{CH}_{3} \mathrm{OH}$ & 0 \\
\hline $\mathrm{H}_{2}^{+}$ & 0 & $\mathrm{e}^{-}$ & $1.30 \times 10^{-4}$ \\
\hline $\mathrm{H}_{3}^{+}$ & 0 & $\perp \mathrm{H}$ & 0 \\
\hline $\mathrm{O}^{3}$ & $2.90 \times 10^{-4}$ & $\perp \mathrm{H}_{\mathrm{c}}$ & 0 \\
\hline $\mathrm{O}^{-}$ & 0 & $\perp \mathrm{H}_{2}$ & 0 \\
\hline $\mathrm{O}^{+}$ & 0 & $\perp \mathrm{O}$ & 0 \\
\hline $\mathrm{O}_{2}$ & 0 & $\perp \mathrm{O}_{2}$ & 0 \\
\hline $\mathrm{C}$ & 0 & $\perp \mathrm{O}_{3}$ & 0 \\
\hline $\mathrm{C}^{-}$ & 0 & $\perp \mathrm{OH}$ & 0 \\
\hline $\mathrm{C}^{+}$ & $1.30 \times 10^{-4}$ & $\perp \mathrm{CO}$ & 0 \\
\hline $\mathrm{OH}$ & 0 & $\perp \mathrm{CO}_{2}$ & 0 \\
\hline $\mathrm{OH}^{+}$ & 0 & $\perp \mathrm{H}_{2} \mathrm{O}$ & 0 \\
\hline $\mathrm{CO}$ & 0 & $\perp \mathrm{HO}_{2}$ & 0 \\
\hline $\mathrm{CO}_{2}$ & 0 & $\perp \mathrm{H}_{2} \mathrm{O}_{2}$ & 0 \\
\hline $\mathrm{H}_{2} \mathrm{O}$ & 0 & $\perp \mathrm{HCO}$ & 0 \\
\hline $\mathrm{H}_{2} \mathrm{O}^{+}$ & 0 & $\perp \mathrm{H}_{2} \mathrm{CO}$ & 0 \\
\hline $\mathrm{H}_{3} \mathrm{O}^{+}$ & 0 & $\perp \mathrm{CH}_{3} \mathrm{O}$ & 0 \\
\hline $\mathrm{HCO}$ & 0 & $\perp \mathrm{CH}_{3} \mathrm{OH}$ & 0 \\
\hline
\end{tabular}

Notes. The symbol $\perp$ denotes a bound/ice species. $\perp \mathrm{H}_{\mathrm{c}}$ is the chemically adsorbed counterpart of $\perp \mathrm{H}$.

and 16 are on dust grains. Our selection of species and their initial abundances (with respect to hydrogen atoms) is given in Table 1.

There are 257 relevant reactions. Gas-phase reactions with the corresponding equations and their coefficients were obtained from the Kinetic Database for Astrochemistry (KiDA; Wakelam et al. 2012). Surface reactions on dust grains were acquired from Cazaux et al. (2010) and comprise 89 reactions (see Appendix A).

\subsection{Chemistry solver}

Chemical reaction rates are solved using a fast and stable semiimplicit scheme, an improved scheme over the first-order backwards differencing (BDF) method developed by Anninos et al. (1997). The derivation of this scheme is given below.

The general expression for implicitly solving ordinary differential rate equations is defined as

$\frac{\mathrm{d} n_{x_{i}}}{\mathrm{~d} t}=C_{x_{i}}-D_{x_{i}}$

where $C_{x_{i}}$ is the creation and $D_{x_{i}}$ is the destruction rate of species $x_{i}$ at future, $t+\mathrm{d} t$, time step. The first-order integration of this differential equation is also known as the backward Euler method. It differs from a forward Euler method in which $C_{x_{i}}$ and $D_{x_{i}}$ would be based on current, known values. Knowing that $D_{x_{i}}$ implicitly depends on the species $x_{i}$ we can submit $D_{x_{i}} \equiv n_{x_{i}} D_{x_{i}}^{\prime}$, with $D_{x_{i}}^{\prime}$ the destruction rate coefficient (Schleicher et al. 2008). Following this, the semi-implicit scheme (Anninos et al. 1997) is obtained by

$$
\begin{aligned}
& \frac{\mathrm{d} n_{x_{i}}}{\mathrm{~d} t}=\frac{n_{x_{i}}^{\text {new }}-n_{x_{i}}^{\text {old }}}{\Delta t}=C_{x_{i}}-n_{x_{i}}^{\text {new }} D_{x_{i}}^{\prime}, \\
& n_{x_{i}}^{\text {new }}=\frac{n_{x_{i}}^{\text {old }}+C_{x_{i}} \Delta t}{1+D_{x_{i}}^{\prime} \Delta t}
\end{aligned}
$$


where old refers to the values at the current time step and new points to the values at $t+\Delta t$, the future time step. Because the new rates, $C_{x_{i}}$ and $D_{x_{i}}^{\prime}$, are also not known at the current time step, which we ideally wish to find, they can be approximated using current, old values through iterating, applying a predictorcorrector method, or by using a mix between old and new values with the values that were previously evaluated. The latter does somewhat depend on the order in which the rates are calculated.

We devised a second-order variant of the semi-implicit method (Eq. (3)) to solve our equations. We applied the trapezoidal rule to integrate our differential equation to gain higher precision. In its derived form, the second-order semi-implicit scheme is presented as

$n_{x_{i}}^{j+1}=\frac{n_{x_{i}}^{j}\left(1-\frac{1}{2} D_{x_{i}}^{\prime j} \Delta t\right)+\frac{1}{2}\left(C_{x_{i}}^{j}+C_{x_{i}}^{j+1}\right) \Delta t}{1+\frac{1}{2} D_{x_{i}}^{\prime j+1} \Delta t}$

where we have replaced the time step descriptor by $j$ and $j+1$ (previously, old and new). This scheme is easily computed while still being an improvement over the explicit schemes and the first-order semi-implicit scheme. The order of error does not drop with respect to a second-order fully implicit method, that is, the local error is $O\left(h^{3}\right)$, the global error $O\left(h^{2}\right)$. Semi-implicit schemes are symplectic integrators in nature and yield far better results than standard Euler methods. We note, however, that for all semi-implicit BDF methods, one must ensure mass conservation, and they are not time reversible.

A higher order BDF method will be more accurate for solving the chemistry, as proven by Bovino et al. (2013), but will also come at a higher computational price. Employing a high time resolution with this scheme is, in our experience, sufficient to have good accuracy while still performing the calculations at an acceptable speed.

\subsection{Gas-phase chemistry}

Gas-phase reactions were acquired from the Kinetic Database for Astrochemistry (Wakelam et al. 2012). We considered every possible reaction (within the scope of the database) that involves our selection of species as given in Table 1.

The 168 gas-phase reactions in our network include bimolecular reactions (e.g., $\mathrm{A}+\mathrm{B} \rightarrow \mathrm{C}+\mathrm{D}$ ), charge-exchange reactions (e.g, $\mathrm{A}^{+}+\mathrm{B} \rightarrow \mathrm{A}+\mathrm{B}^{+}$), radiative associations (e.g., $\mathrm{A}+\mathrm{B} \rightarrow$ $\mathrm{AB}+$ photon), associative detachment (e.g., $\mathrm{A}^{-}+\mathrm{B} \rightarrow \mathrm{AB}+$ $\mathrm{e}^{-}$), electronic recombination and attachment (e.g., $\mathrm{AB}^{+}+\mathrm{e}^{-} \rightarrow$ $A+B)$, ionization or dissociation of neutral species by UV photons, ionization or dissociation of species by direct collision with cosmic-ray particles or by secondary UV photons following $\mathrm{H}_{2}$ excitation.

\subsection{Dust chemistry}

The solid-phase reactions or the reaction rate coefficients were gathered from Cazaux et al. (2010). We have included several additional reactions in this work. The grain surface rate equations were simplified and set in easily accessible forms. To do this, we unified all the different reactions involving dust grains into five equation types. These are (A) adsorption of gasphase species onto dust surfaces; (B) thermal desorption of ices; (C) two-body reactions on grain surfaces including chemical desorption; (D) cosmic-ray processes; and (E) photo-processes with UV photons that include photodissociation and photodesorption. The equations are explained in detail in the following five subsections.

\subsubsection{Adsorption onto dust grains}

Species in the gas phase can be accreted onto grain surfaces. This depends on the motion of the gas species relative to the dust particle. Since the motions are dominated by thermal velocity, the adsorption rate depends on the square root of the gas temperature as $v_{\text {th }}=\sqrt{8 k_{\mathrm{B}} T_{\mathrm{g}} / \pi m}$. Once the gas species is in contact with the dust, there is a probability for it to stick on the surface of the grain. The sticking coefficient is calculated as

$S(T)=\left(1+0.4\left(\frac{T_{\mathrm{g}}+T_{\mathrm{d}}}{100}\right)^{0.5}+0.2 \frac{T_{\mathrm{g}}}{100}+0.08\left(\frac{T_{\mathrm{g}}}{100}\right)^{2}\right)^{-1}$,

where $T_{\mathrm{g}}$ is the gas temperature and $T_{\mathrm{d}}$ is the dust temperature (Hollenbach \& McKee 1979). We note that this coefficient is based on $\mathrm{H}$ atoms. Using this, the adsorption rate can be formulated as,

$k_{\mathrm{ads}}=n_{\mathrm{d}} \sigma_{\mathrm{d}} v_{x_{i}} \quad \mathrm{~s}^{-1}$,

$R_{\mathrm{ads}}=n_{x_{i}} k_{\mathrm{ads}} S(T) \mathrm{cm}^{-3} \mathrm{~s}^{-1}$,

where $k_{\text {ads }}$ is the adsorption rate coefficient, $R_{\text {ads }}$ is the adsorption rate, $n_{\mathrm{d}}$ is the number density of dust grains, $\sigma_{\mathrm{d}}$ is the cross section of the grain, $v_{x_{i}}$ is the thermal velocity of species $x_{i}$, that is, $v_{x_{i}}=\sqrt{8 k_{\mathrm{B}} T_{\mathrm{g}} / \pi m_{x_{i}}}$, with $m_{x_{i}}$ the mass in grams, and $n_{x_{i}}$ is the number density of the engaging species. In this equation, $n_{\mathrm{d}} \sigma_{\mathrm{d}}$ represents the total cross section of dust in $\mathrm{cm}^{-1}$, which is obtained by integrating over the grain-size distribution. We adopted the grain-size distribution of Mathis et al. (1977), from here on MRN, with a value of $\alpha_{\mathrm{MRN}}=\left\langle n_{\mathrm{d}} \sigma_{\mathrm{d}} / n_{\mathrm{H}}\right\rangle_{\mathrm{MRN}}=1 \times 10^{-21} \mathrm{~cm}^{2}$. We chose this distribution rather than the one of Weingartner \& Draine (2001), from here on WD, which has a total cross section larger by a factor three, that is, $\alpha_{\mathrm{WD}}=3 \times 10^{-21} \mathrm{~cm}^{2}$, because MRN did not include poly-cyclic aromatic hydrocarbons (PAHs). The freeze-out of species on PAHs to form ices is not known.

\subsubsection{Thermal desorption, evaporation}

After species are bound on grain surfaces, they can evaporate back into the gas. The evaporation rate depends exponentially on the dust temperature and on the binding energies of the species with the substrate. The binding energy of each species differs according to the type of substrate. We consider two possible substrates in this study, bare surfaces (assuming carbon substrate) and water ice substrate, since ices are mostly made of water. Species adsorbed on water ice substrate have in most cases binding energies higher than on bare dust or other ices (e.g., see Cuppen \& Herbst 2007). Species on top of CO, which can attain a significant coverage on dust, have binding energies that more closely resemble the binding energies of bare dust (e.g., Sandford \& Allamandola 1988; Karssemeijer et al. 2014). Therefore, in this study we consider the binding energies on $\mathrm{CO}$ to be similar to those of bare dust.

We computed the fraction of the dust covered by (water) ice $\mathcal{F}_{\text {ice }}$ and bare $\mathcal{F}_{\text {bare }}$ to distinguish between the two. Together with the deposited amount of water ice, this fraction depends on the total number of possible attachable sites on grain surfaces per cubic cm of space, designated as $n_{\mathrm{d}} n_{\text {sites }}$, which is defined as

$n_{\mathrm{d}} n_{\text {sites }}=n_{\mathrm{d}} \frac{4 \pi r_{\mathrm{d}}^{2}}{a_{\mathrm{pp}}^{2}}=n_{\mathrm{d}} \sigma_{\mathrm{d}} \frac{4}{a_{\mathrm{pp}}^{2}} \simeq 4.44 \times 10^{-6} n_{\mathrm{H}} \mathrm{cm}^{-3} \mathrm{mly}^{-1}$,

where the radius of dust is given by $r_{\mathrm{d}}$ and the typical separation between two adsorption sites on a grain surface is given 
Table 2. Binding energies for the substrates bare grain and water ice.

\begin{tabular}{|c|c|c|c|c|c|}
\hline \multicolumn{4}{|c|}{ Substrate } & \multicolumn{2}{|c|}{ Substrate } \\
\hline Species & Bare $(\mathrm{K})$ & Ice $(\mathrm{K})$ & Species & Bare $(\mathrm{K})$ & Ice $(\mathrm{K})$ \\
\hline$\perp \mathrm{H}$ & $500^{e, f}$ & $650^{d}$ & $\perp \mathrm{CO}_{2}$ & $2300^{g}$ & $2300^{g}$ \\
\hline$\perp \mathrm{H}_{\mathrm{c}}$ & $10000^{c}$ & $10000^{c}$ & $\perp \mathrm{H}_{2} \mathrm{O}$ & $4800^{a, h}$ & $4800^{a, h}$ \\
\hline$\perp \mathrm{H}_{2}$ & $300^{i}$ & $300^{i}$ & $\perp \mathrm{HO}_{2}$ & $4000^{i}$ & $4300^{d}$ \\
\hline$\perp \mathrm{O}$ & $1700^{i}$ & $1700^{i}$ & $\perp \mathrm{H}_{2} \mathrm{O}_{2}$ & $6000^{i}$ & $5000^{d}$ \\
\hline$\perp \mathrm{O}_{2}$ & $1250^{i}$ & $900^{d, g}$ & $\perp \mathrm{HCO}$ & $1100^{l}$ & $3100^{k}$ \\
\hline$\perp \mathrm{O}_{3}$ & $2100^{i}$ & $1800^{d}$ & $\perp \mathrm{H}_{2} \mathrm{CO}$ & $1100^{l}$ & $3100^{h}$ \\
\hline$\perp \mathrm{OH}$ & $1360^{d}$ & $3500^{d}$ & $\perp \mathrm{CH}_{3} \mathrm{O}$ & $1100^{l}$ & $3100^{k}$ \\
\hline$\perp \mathrm{CO}$ & $1100^{b, f}$ & $1300^{g, j}$ & $\perp \mathrm{CH}_{3} \mathrm{OH}$ & $1100^{l}$ & $3100^{k}$ \\
\hline
\end{tabular}

Notes. ${ }^{(a)}$ Sandford \& Allamandola (1988); ${ }^{(b)}$ Collings et al. (2003); ${ }^{(c)}$ Cazaux \& Tielens (2004); ${ }^{(d)}$ Cuppen \& Herbst (2007); ${ }^{(e)}$ Cazaux et al. (2010); ${ }^{(f)}$ Garrod \& Pauly (2011); ${ }^{(g)}$ Noble et al. (2012a); (h) Noble et al. (2012b); ${ }^{(i)}$ Dulieu et al. (2013), with updated $\perp \mathrm{O}$ binding energies; ${ }^{(j)}$ Karssemeijer et al. (2014); ${ }^{(k)}$ we relate these to $\mathrm{H}_{2} \mathrm{CO}^{i}$ binding energies; ${ }^{(l)}$ we relate these to $\mathrm{CO}^{\text {bg }}$ binding energies.

by $a_{\mathrm{pp}}$, which we assume to be $3 \AA$. A full monolayer (mly) is reached when all the possible sites on a grain surface are occupied by an atom or a molecule. To convert this from monolayers to number densities, one needs to multiply by $n_{\mathrm{d}} n_{\text {sites. }}$. For the dust-to-gas mass ratio $\epsilon_{\mathrm{d}}$, intrinsic to the dust number density $n_{\mathrm{d}}$, we assumed the typical value of $\epsilon_{\mathrm{d}}=0.01$.

We obtained the fractions, $\mathcal{F}_{\text {ice }}$ and $\mathcal{F}_{\text {bare }}$, in the following manner: if the grain surface is covered by less than 1 mly of water ice,

$\mathcal{F}_{\text {ice }}=\frac{n_{\perp \mathrm{H}_{2} \mathrm{O}}}{n_{\mathrm{d}} n_{\text {sites }}}$.

When the grain is covered by more than 1 layer of water ice, $\mathcal{F}_{\text {ice }}=1$. The bare fraction of the dust is obtained by

$\mathcal{F}_{\text {bare }}=1-\mathcal{F}_{\text {ice }}$.

We note that at this stage we assumed that the bound species are homogeneously distributed and neglected the cases where stratified layers of species can form. This assumption can lead to an over- or underestimation of reaction rates because the species abundances on different substrates can differ. We expect, however, that since the situation $\mathcal{F}_{\text {bare }} \sim \mathcal{F}_{\text {ice }}$ is not very common, the over- or underestimation will be marginal. With these definitions, we can formulate the evaporation rate as follows:

$$
\begin{aligned}
& k_{\text {evap }}=v_{0}\left(\mathcal{F}_{\text {bare }} \exp \left(-\frac{E_{\text {bare }, i}}{T_{\mathrm{d}}}\right)+\mathcal{F}_{\text {ice }} \exp \left(-\frac{E_{\text {ice }, i}}{T_{\mathrm{d}}}\right)\right) \mathrm{s}^{-1}, \\
& R_{\text {evap }}=n_{x_{i}} k_{\text {evap }} \mathrm{cm}^{-3} \mathrm{~s}^{-1},
\end{aligned}
$$

where $k_{\text {evap }}$ is the evaporation rate coefficient, $R_{\text {evap }}$ is the evaporation rate, $v_{0}$ is the oscillation frequency, which is typically $10^{12} \mathrm{~s}^{-1}$ for physisorbed species, $E_{\mathrm{bare}, i}$ and $E_{\mathrm{ice}, i}$ are the binding energies of species $x_{i}$ on bare grains and ices. The species specific binding energies can be found in Table 2 .

\subsubsection{Two-body reactions on dust grains}

While species are attached to grain surfaces, they can move around by thermal diffusion and meet other species with which they can react to form new molecules. The mobility of the species depends on the oscillation frequency $v_{0}$. We only consider physisorbed species in this work, except for neutral hydrogen, where we also take chemisorption into account. In addition to the mobility, the reaction rate depends on the specific binding energy with the substrate and on the dust temperature. When two species meet, they can immediately react if there is no (or little) reaction barrier. If the reaction barrier is high, however, it might be crossed by tunneling. The probability of overcoming the reaction barrier by tunneling is given by

$P_{\text {reac }}=\exp \left(-(2 a / \hbar) \sqrt{2 m_{\text {red }} k_{\mathrm{B}} E_{\mathrm{a}}}\right)$,

where $a=1 \AA, m_{\text {red }}$ is the reduced mass of the two engaging species, that is, $m_{\text {red }}=\left(m_{i} \times m_{j}\right) /\left(m_{i}+m_{j}\right), h$ is the Planck constant, and $E_{\mathrm{a}}$ is the energy of the barrier needed for the reaction to occur. The probability is $P_{\text {reac }}=1$ if there is no barrier for the reaction to take place. We did not consider the reaction diffusion competition (Garrod \& Pauly 2011). If this were included, the tunneling probabilities would become much higher.

For $\mathrm{CO}$ hydrogenation reactions that have a barrier, that is, $\perp \mathrm{H}+\perp \mathrm{CO}$ and $\perp \mathrm{H}+\perp \mathrm{H}_{2} \mathrm{CO}$, we deduced an "effective" tunneling barrier energy from the reaction rates obtained by Atkinson et al. (2004) and Fuchs et al. (2009) at low ( 10 K) temperatures. The activation barriers we calculated are $600 \mathrm{~K}$ and $400 \mathrm{~K}$, respectively. The height of these barriers are verified from recent experimental results (Minissale et al. priv. comm.). More precise measurements are being performed. The used activation barriers can be found in Appendix A.

When a reaction occurs, the product either remains on the surface or immediately desorbs into the gas phase because of the exothermicity of the reaction. The probabilities of desorption are given by $\delta_{\text {bare }}$ and $\delta_{\text {ice }}$ for our two substrates. The fraction that remains on the surface will be $1-\delta$. Non-exothermic reactions that do not desorb are by definition mutiplied by 1 . With this information, we formulate the two-body reaction rate on grain surfaces as

$$
\begin{aligned}
k_{2 \text { body }}= & v_{0} \mathcal{F}_{\text {bare }}\left(\exp \left(-\frac{2}{3} \frac{E_{\text {bare }, i}}{T_{\mathrm{d}}}\right)+\exp \left(-\frac{2}{3} \frac{E_{\text {bare }, j}}{T_{\mathrm{d}}}\right)\right)\left(1-\delta_{\text {bare }}\right) \\
& +v_{0} \mathcal{F}_{\text {ice }}\left(\exp \left(-\frac{2}{3} \frac{E_{\text {ice }, i}}{T_{\mathrm{d}}}\right)+\exp \left(-\frac{2}{3} \frac{E_{\text {ice }, j}}{T_{\mathrm{d}}}\right)\right)\left(1-\delta_{\text {ice }}\right) \\
R_{\text {body }}= & \frac{n_{x_{i}} n_{x_{j}}}{n_{\mathrm{d}} n_{\text {sites }}} P_{\text {reac }} k_{2 \text { body }} \mathrm{cm}^{-3} \mathrm{~s}^{-1}
\end{aligned}
$$

where $k_{2 \text { body }}$ is the two-body rate coefficient, $R_{2 \text { body }}$ is the twobody reaction rate. The exponent in this equation represents the diffusion of species on the surface, and we assume that diffusion occurs with a barrier of two-thirds of the binding energy (67\% Dulieu et al. 2013; 40\% Collings et al. 2003; 90\% Barzel $\&$ Biham 2007). The desorption rate is obtained from the complement of $1 \delta$ of the rate coefficient.

The desorption probabilities $\delta_{\text {bare }}$ for the exothermic reactions $\perp \mathrm{H}+\perp \mathrm{O} \rightarrow \mathrm{OH}, \perp \mathrm{H}+\perp \mathrm{OH} \rightarrow \mathrm{H}_{2} \mathrm{O}, \perp \mathrm{O}+\perp \mathrm{O} \rightarrow \mathrm{O}_{2}$ were adopted from Dulieu et al. (2013) and are $\delta_{\text {bare }}=0.5,0.9,0.6$. For the hydrogenation reactions $\perp \mathrm{HCO}+\perp \mathrm{H} \rightarrow \mathrm{H}_{2} \mathrm{CO}$ and $\perp \mathrm{CH}_{3} \mathrm{O}+\perp \mathrm{H} \rightarrow \mathrm{CH}_{3} \mathrm{OH}$, we assumed a desorption fraction of $\delta_{\text {bare }}=5 \%$ and for $\perp \mathrm{H}_{2} \mathrm{CO}+\perp \mathrm{H} \rightarrow \mathrm{CH}_{3} \mathrm{O} \delta_{\text {bare }}=50 \%$. In our next paper, we will report the different chemical desorption yields on different types of surfaces, estimated by laboratory experiments (Cazaux et al., in prep.). For the desorption probabilities on icy substrates $\delta_{\text {ice }}$, we considered that chemical desorption is much weaker than on bare surfaces and assumed that $\delta_{\text {ice }}=\delta_{\text {bare }} / 5$ (deduced from Dulieu et al. 2013). We did not take into account that chemical desorption has a dependence on surface coverage (Minissale \& Dulieu 2014). 


\subsubsection{Cosmic-ray processes on grain surfaces}

Cosmic-ray reaction rates on grain surfaces are assumed to be the same as the rates found in the gas phase. We included several of the cosmic-ray reactions for surface bound species. Cosmicray processes are usually inefficient destruction mechanisms, but can dominate the destruction rates deep inside the cloud. These reaction rates depend on the cosmic-ray ionization rate per $\mathrm{H}_{2}$ molecule, which we adopted as $\zeta_{\mathrm{H}_{2}}=5 \times 10^{-17} \mathrm{~s}^{-1}$ (Indriolo et al. 2007; Hocuk \& Spaans 2011; Chaparro Molano \& Kamp 2012). The cosmic-ray reaction rate is formulated as

$k_{\mathrm{CR}}=z_{x_{i}} \zeta_{\mathrm{H}_{2}} \mathrm{~s}^{-1}$,

$R_{\mathrm{CR}}=n_{x_{i}} k_{\mathrm{CR}} \mathrm{cm}^{-3} \mathrm{~s}^{-1}$,

where $k_{\mathrm{CR}}$ is the cosmic-ray rate coefficient, $R_{\mathrm{CR}}$ is the cosmicray reaction rate, and $z_{x_{i}}$ the cosmic-ray ionization rate factor that is subject to the ionizing element (see KiDA database).

Cosmic-ray-induced UV (CRUV) photons were also considered within the same equation (Eq. (13)). In this case, $z_{x_{i}}$ is replaced by $z_{\mathrm{CRUV}}$, the UV photon generation rate per cosmic-ray ionization. UV photons from cosmic rays do not suffer from radiation attenuation as normal UV photons do. Hence, the lack of dependence on optical depth in this formula.

\subsubsection{Photo-processes on grain surfaces}

When UV photons arrive on a dust particle, they can interact with the adsorbed species and either photodissociate or photoevaporate them. We used the same formula for both types of photo-processes. Photoreactions scale linearly with the local radiation flux $\left(\mathrm{erg} \mathrm{cm}^{-2} \mathrm{~s}^{-1}\right)$. The radiation field strength is necessarily a function of extinction, which is given by $\xi_{x_{i}} A_{V}$, where $\xi_{x_{i}}$ is the extinction factor that is contingent on the relevant species. We obtain $A_{V}$ by dividing the column density $N_{\mathrm{H}}$ over the scaling factor, that is, $A_{V}=N_{\mathrm{H}} / 2.21 \times 10^{21} \mathrm{mag}$ (Güver \& Özel 2009). The column densities were computed by integrating the density from the simulation boundaries to each point. Simply, this is $N_{\mathrm{H}}=\Sigma_{i} n_{\mathrm{H}} \mathrm{d} s_{i}$, with $\mathrm{d} s_{i}$ being the path length of the smallest resolution element in which the density remains constant. For this purpose, we constructed a ray-tracing algorithm using 14 equally weighted rays with long characteristics (traveling from inside to outside). The ray separation is set by our highest resolution. We assumed an isotropic UV radiation field with a flux of $1 G_{0}$, where $G_{0} \equiv 1.6 \times 10^{-3} \mathrm{erg} \mathrm{cm}^{-2} \mathrm{~s}^{-1}$. The general photo-process rate equations are defined as

$k_{\text {phot }}=\alpha_{x_{i}} \mathrm{e}^{-\xi_{x_{i}} A_{V}} \mathrm{~s}^{-1}$,

$R_{\mathrm{phot}}=n_{x_{i}} f_{\mathrm{ss}} k_{\mathrm{phot}} F_{\mathrm{UV}} \mathrm{cm}^{-3} \mathrm{~s}^{-1}$.

where $k_{\text {phot }}$ is the photo-process rate coefficient, $R_{\text {phot }}$ is the photo-process reaction rate, $\alpha_{x_{i}}$ is the unattenuated rate coefficient, $f_{\mathrm{ss}}$ is the self-shielding factor, and $F_{\mathrm{UV}}$ is the UV flux in units of $1.71 G_{0}$, that is, $G_{0}=1$ gives $F_{\mathrm{UV}}=0.58$. The factor 1.71 arises from the conversion from the often used Draine field (Draine 1978) to the Habing field for the far-ultraviolet (FUV) intensity. We used the same $\alpha_{x_{i}}, \xi_{x_{i}}$, and $f_{\mathrm{ss}}$ for the gas phase as we did for the surface reactions.

When UV photons arrive on an icy surface with multiple layers, we only allowed the first two layers to be penetrated by UV photons. As shown by Andersson et al. (2006), Arasa et al. (2010), and Muñoz Caro et al. (2010), only the uppermost few layers contribute to photodesorption. Photodissociation does seem to occur deeper into the ice, but trapping and recombination of species tend to dominate (Andersson \& van Dishoeck 2008). This means that the highest number density that the photons can see is $n_{x_{i}}=$ $\min \left(n_{x_{i}}, 2 n_{\mathrm{d}} n_{\text {sites }}\right)$. This restriction is also enforced for reactions with CRUV photons, as given in Sect. 3.3.4.

Self-shielding, denoted as $f_{\text {ss }}$, was taken into account for $\mathrm{H}_{2}$ and $\mathrm{CO}$ molecules. These molecules can shield the medium against photo-processes on grain surfaces as well as for species in the gas phase. The self-shielding factor for $\mathrm{H}_{2}$ was obtained from Draine \& Bertoldi (1996), Eq. (37), which is formulated as

$f_{\mathrm{ss}}=\frac{0.965}{\left(1+x / b_{5}\right)^{2}}+\frac{0.035}{(1+x)^{0.5}} \times \exp \left[-8.5 \times 10^{-4}(1+x)^{0.5}\right]$,

where $x \equiv N_{\mathrm{H}_{2}} / 5 \times 10^{14} \mathrm{~cm}^{-2}, b_{5} \equiv b / 10^{5} \mathrm{~cm} \mathrm{~s}^{-1}$, and $b$ is the line broadening of $\mathrm{H}_{2}$ lines, which we take as $3 \mathrm{~km} \mathrm{~s}^{-1}$. This factor is only a function of $\mathrm{H}_{2}$ column density $N_{\mathrm{H}_{2}}$. We used the column density algorithm to compute the $\mathrm{H}_{2}$ column for this purpose. For $\mathrm{CO}$ molecules, self-shielding was achieved by incorporating the self-shielding tables from Visser et al. (2009) into our code. Given an $\mathrm{H}_{2}$ column and a $\mathrm{CO}$ column, the factor $f_{\mathrm{ss}}$ was acquired from the tables. For all other species we took $f_{\mathrm{ss}}=1$.

Photodesorption is only implemented here for $\perp \mathrm{CO}$ molecules. Since CO molecules in the gas phase can strongly affect the thermal balance of collapsing molecular clouds, as we have shown in an earlier work (Hocuk et al. 2014), detailed processes were taken into account to correctly determine gas-phase abundances of $\mathrm{CO}$. This encompasses both the normal and the CRUV photons. Photodesorption is not expected to be the dominant destruction mechanism of $\perp \mathrm{CO}$, but is a route to desorb surface-bound $\mathrm{CO}$ molecules directly into the gas phase. The two implemented reactions are

$$
\begin{aligned}
& \perp \mathrm{CO}+\mathrm{UV} \text { photon } \rightarrow \mathrm{CO}, \\
& \perp \mathrm{CO}+\text { CRUV photon } \rightarrow \mathrm{CO} .
\end{aligned}
$$

For these two photo-chemical reactions $\alpha_{x_{i}}$ of Eq. (15) represents the number of $\mathrm{CO}$ photodesorptions per second per unit radiation flux (Hollenbach et al. 2009; Chaparro Molano \& Kamp 2012). This variable depends on the photodesorption yield, which was experimentally obtained and adopted by us as $Y_{\mathrm{CO}}=1.0 \times 10^{-2}$ (Fayolle et al. 2011). With respect to previous values (Öberg et al. 2009), these yields are higher by about a factor 4 . In addition, only the first two monolayers were assumed to be penetrable by UV photons for these rates.

\section{Analytical method: thermodynamics}

To address the thermodynamics of the gas cloud, we calculated time-dependent heating and cooling rates that complemented the chemistry calculations. In this way, we obtained the gas and dust temperatures by solving the thermal balance.

\subsection{Heating and cooling}

We included the most prominent heating processes that are relevant to our work. The non-equilibrium heating processes include

- photoelectric heating;

- $\mathrm{H}_{2}$ photodissociation heating;

- $\mathrm{H}_{2}$ collisional de-excitation heating;

- cosmic-ray heating;

- gas-grain collisional heating (when $T_{\text {gas }}<T_{\text {dust }}$ ). 
Compressional heating and shock heating are by default taken into account through the hydrodynamics, the EOS, and the shock-detection routines of our code, FLASH.

The cooling of the gas is ensured by several different types of non-equilibrium processes. These processes are

- electron recombination with PAHs cooling;

- electron impact with H (i.e., Ly- $\alpha$ ) cooling;

- metastable transition of [OI]-630 nm cooling;

- fine-structure line cooling of [OI]-63 $\mu \mathrm{m}$ and [CII]-158 $\mu \mathrm{m}$;

- molecular ro-vibrational cooling by $\mathrm{H}_{2}, \mathrm{CO}, \mathrm{OH}$, and $\mathrm{H}_{2} \mathrm{O}$;

- and gas-grain collisional cooling (when $T_{\text {gas }}>T_{\text {dust }}$ ).

Cooling by adiabatic expansion was, again, handled by the standard EOS routines of FLASH. The heating and cooling functions and their rates are described in Hocuk et al. (2014).

\subsection{Dust temperature}

The dust temperature is a crucial parameter that not only influences the gas temperature through the heating and the cooling rates, but also affects the chemical reaction rates. This eventually drives the formation and build-up of ices. We follow Hollenbach et al. (1991), also mentioned in Latif et al. (2012), but with some adaptations of our own. The initial estimate of the dust temperature, $T_{\mathrm{d}, i}$, which incorporates the attenuated incident UV radiation field, the cosmic microwave background temperature $T_{\mathrm{CMB}}=2.725 \mathrm{~K}$, and the infrared dust emission, is defined as

$$
\begin{aligned}
T_{\mathrm{d}, i}^{5}= & 8.9 \times 10^{-11} v_{0} G_{0} \exp \left(-1.8 A_{V}\right)+T_{\mathrm{CMB}}^{5}+3.4 \times 10^{-2} \\
& \times\left[0.42-\ln \left(3.5 \times 10^{-2} \tau_{100} T_{0}\right)\right] \tau_{100} T_{0}^{6},
\end{aligned}
$$

where the adopted value $v_{0}=3 \times 10^{15} \mathrm{~s}^{-1}$ represents the most efficient absorbing frequency over the visual and UV wavelengths, $\tau_{100}$ is the emission optical depth at $100 \mu \mathrm{m}$, and $T_{0}$ is the equilibrium dust temperature at the cloud surface due to unattenuated incident FUV field alone (Hollenbach et al. 1991). $T_{0}$ in this case equates to $T_{0}=12.17 G_{0}^{1 / 5} \mathrm{~K}$. If it is assumed that the incident FUV flux equals the outgoing flux of dust radiation from $T_{0}$, then $\tau_{100}=2.7 \times 10^{3} G_{0} T_{0}^{-5}$ (Hollenbach et al. 1991). Knowing $T_{0}$ fixes $\tau_{100}$ to a value of 0.001 . This makes it independent of optical depth. We display this by plotting the dust temperature as a function of visual extinction in Fig. 2, which also shows other dust temperature calculations.

To accommodate for the depth dependence of the infrared emission, we calculated $\tau_{100}$ in a different fashion. Since $A_{V}=$ $2.5 \log _{10}(e) \tau_{V} \simeq 1.086 \tau_{V}$, where $\tau_{V}$ is the opacity at optical (predominantly $550 \mathrm{~nm}$ ) wavelengths, and because

$\tau_{\lambda}=\tau_{100}\left(\frac{100 \mu \mathrm{m}}{\lambda}\right)^{\beta_{\text {sed }}}$,

with $\lambda$ being the wavelength and $\beta_{\text {sed }}$ the sub-mm slope of the spectral energy distribution known as the spectral emissivity index, we can rewrite $\tau_{100}$ as a function of $A_{V}$ in the form

$\tau_{100}=\tau_{550 \mathrm{~nm}}\left(\frac{550 \mathrm{~nm}}{100 \mu \mathrm{m}}\right)^{1.3}=9.4 \times 10^{-4} A_{V}$.

For $\beta_{\text {sed }}$ we take the value of 1.3 , which gives the same $\tau_{100}$ at $A_{V} \simeq 1$ as Hollenbach et al. (1991) advocated. A spectral emissivity index between $\beta_{\text {sed }}=1-2$ is typically found for the Milky Way (Miettinen et al. 2012; Arab et al. 2012). However, we now have a higher value of $\tau_{100}$ at higher $A_{V}$ to account for the depth dependence.

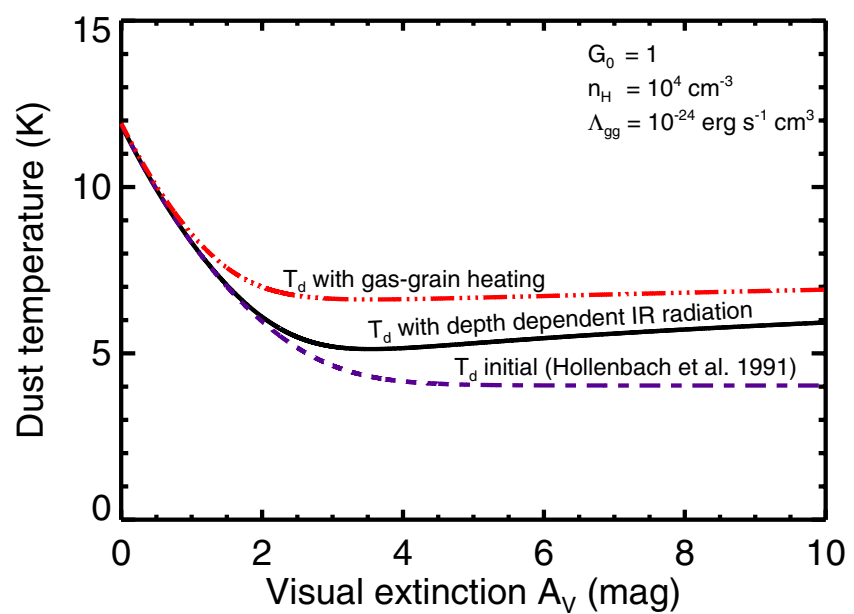

Fig. 2. Dust temperature estimated by including additional heating sources. The purple dashed line displays the initial estimate of the dust temperature as given by Hollenbach et al. (1991). The black solid line is the dust temperature that takes into account the depth dependence of infrared radiation by using a simple scaling with opacity. The red dot-dashed line also considers the gas-grain heating for which the variables $\Lambda_{\mathrm{gg}}$ and $n_{\mathrm{H}}$ are fixed here to serve in this example.

We also considered the heating of dust grains by the gas through the gas-grain collisional heat exchange. Since dust grains have a larger heat capacity, the heating of dust grains will be considerably weaker than the cooling of the gas, but this process might still slightly increase the dust temperature. Assuming that there is a temperature equilibrium in which the equilibrium timescale is much shorter than a free-fall time, the energy balance to reach a stable dust temperature is given by

$\Lambda_{\mathrm{gg}}=\Gamma_{\mathrm{rad}}$,

where $\Lambda_{\text {gg }}$ is the gas-grain collisional heating and $\Gamma_{\text {rad }}$ is the radiative losses due to blackbody radiation. Note that these are the losses from raising the dust temperature to a higher value by gasgrain collisional heat exchange than the equilibrium temperature given in Eq. (16). The losses can be described as

$\Gamma_{\text {rad }}=4 \sigma_{\mathrm{SB}}\left(T_{\mathrm{d}}^{4}-T_{\mathrm{d}, i}^{4}\right) \rho_{\mathrm{d}} \kappa_{\mathrm{P}} \operatorname{erg~cm}^{-3} \mathrm{~s}^{-1}$,

with $\sigma_{\mathrm{SB}}=5.67 \times 10^{-5} \mathrm{erg} \mathrm{cm}^{-2} \mathrm{~s}^{-1} \mathrm{~K}^{-4}$ the Stefan-Boltzmann constant, $\rho_{\mathrm{d}}$ the mass density of dust, and $\kappa_{\mathrm{P}}$ the Planck mean opacity. Here, we also make use of our initial estimate of the dust temperature $T_{\mathrm{d}, i}$ and expect $T_{\mathrm{d}, i}$ to be at a stable equilibrium dust temperature when there is no additional heating source. We adopt the relation

$\kappa_{\mathrm{P}}=4 \times 10^{-4} T_{\mathrm{d}}^{2} \mathrm{~cm}^{2} \mathrm{~g}^{-1}$

as presented by Omukai (2000) for a typical molecular cloud composition (Pollack et al. 1994) and for $T_{\mathrm{d}} \lesssim 50 \mathrm{~K}$, but we did not consider Rosseland mean opacity in our solution (Stamatellos et al. 2007; Dopcke et al. 2011). Combining Eqs. (19) and (20), we obtain

$T_{\mathrm{d}}^{4}=\frac{\Lambda_{\mathrm{gg}}}{4 \sigma_{\mathrm{SB}} \rho_{\mathrm{d}} \kappa_{\mathrm{P}}}+T_{\mathrm{d}, i}^{4}$.

Replacing $\kappa_{\mathrm{P}}$ with Eq. (21) and by using $\rho_{\mathrm{d}}=\epsilon_{\mathrm{d}} \rho$ for the dust mass density, with $\epsilon_{\mathrm{d}}=0.01$, we can formulate

$T_{\mathrm{d}}^{6}=\frac{\Lambda_{\mathrm{gg}}\left(T_{\mathrm{d} *}\right)}{9.072 \times 10^{-10} \rho}+T_{\mathrm{d}, i}^{4} T_{\mathrm{d} *}^{2}$, 


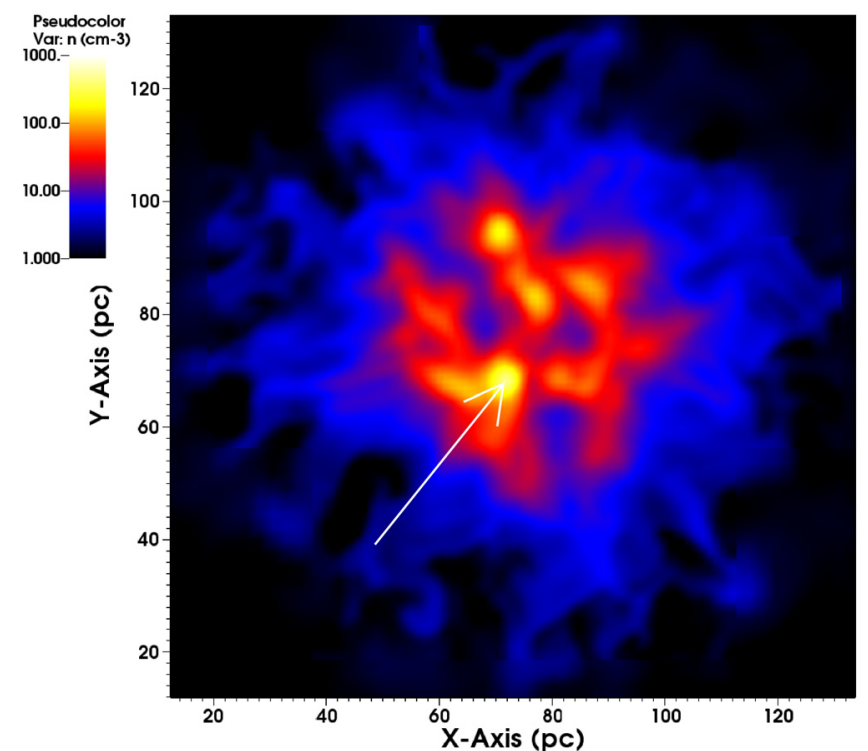

Fig. 3. Clumpy structure of a translucent cloud. Density slice along the $Z$-axis of the evolving cloud at $t=1.27 \times 10^{7} \mathrm{yr}$ after initiation. The white arrow indicates the dense clump that is used for the results in this work.

where $T_{\mathrm{d} *}$ is a prediction for the real dust temperature. This socalled sextic equation is a transcendental equation and unsolvable analytically for $T_{\mathrm{d} *}=T_{\mathrm{d}}$, but we can solve it numerically by way of iteration while taking the first-order estimate of the dust temperature as $T_{\mathrm{d} *}=T_{\mathrm{d}, i}$.

In the end, we have a slightly higher, more accurate dust temperature than our initial estimate that increases with density as a result of better gas-grain coupling, and with optical depth for infrared radiation. To highlight the difference in dust temperature by our adjustments, the three different dust temperatures are displayed together in Fig. 2.

\section{Results}

When we started our simulation, the turbulence created a filamentary structure of the gas inside the cloud. It slowly evolved into a more clumpy structure with dense clumps that were gravitationally unstable. Ten million years after the initial phases, we chose the densest part of our cloud, a collapsing clump of radius $r_{\text {clump }}=1 \mathrm{pc}$ to follow its evolution. The density of our clump grew from $n_{\mathrm{H}} \sim 10^{2}$ to $10^{4.5} \mathrm{~cm}^{-3}$ during this time. The clump was located close to the cloud center. In Fig. 3 we display this clump after nearly 13 Myr of simulation.

To investigate how the chemistry adapts, we recorded the species abundances, their formation rates, and the thermodynamic quantities in time and in space. The results we report as a function of time are based on the mean value of the cells located inside the chosen clump. We also present plots of the clump conditions pertaining to a region of space at a fixed time. In this case, we show the growth of ice layers as a function of visual extinction and present maps in Cartesian coordinates. To also expose the outer regions, we then examine a greater radial distance, with $r_{\text {map }}=5 \mathrm{pc}$.

\subsection{Evolution of the clump}

The density of the clump after 10 million years of cloud evolution is $n_{\mathrm{H}}=200 \mathrm{~cm}^{-3}$, corresponding to an $A_{V}$ of near unity.

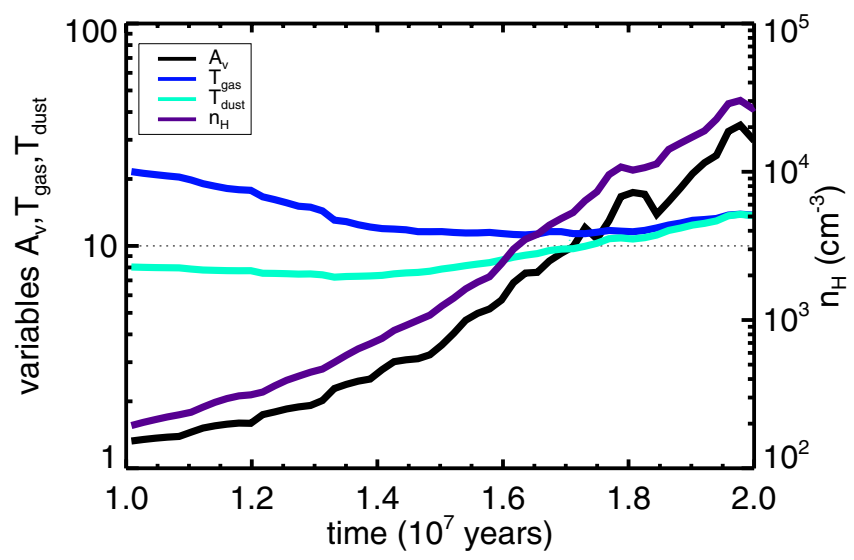

Fig. 4. Time evolution of clump parameters. The variables $A_{V}$ (black), $T_{\text {gas }}$ (blue), $T_{\text {dust }}$ (light blue), and $n_{\mathrm{H}}$ (purple) are plotted as a function of dynamical time.

This increases to a $3 \times 10^{4} \mathrm{~cm}^{-3}$ after another 10 million years, see Fig. 4. Both the gas temperature and the dust temperature of the clump within the same time interval (10-20 Myr) initially decrease as the clump becomes denser, and they rise as a result of compressional heating after rapid collapse sets in. At densities of $n_{\mathrm{H}}=2 \times 10^{4} \mathrm{~cm}^{-3}$ and above, gas-dust coupling allows the two physical states to enjoy the same temperature, with $T \gtrsim 12 \mathrm{~K}$. This occurs around an $A_{V}$ of 20. The optical depth is high because the clump is deeply embedded inside the cloud, close to its center. The collapse of our clump is not delayed, nor does it fragment during the course of the simulation.

\subsection{Time evolution of species abundances}

We plot in Fig. 5 the time evolution of the species abundances in the gas phase and on grain surfaces. These are the results of the abundances within our 1 pc clump.

\section{Gas-phase species abundances}

In the upper panel of Fig. 5, where we plot the gas-phase species, the amount of ionized carbon decreases with passing time and as the cloud becomes denser, while in the meantime the neutral carbon abundance increases. Carbon is readily converted into $\mathrm{CO}$ when the cloud enters a translucent stage at the clump density of $n_{\mathrm{H}}=10^{3} \mathrm{~cm}^{-3}$ and at $A_{V}=3$. CO peaks at $t=$ $1.6 \times 10^{7} \mathrm{yr}$, with a peak abundance of $n_{\mathrm{CO}} / n_{\mathrm{H}}=1.28 \times 10^{-4}$, which amounts to $99 \%$ of all the carbon. This occurs at a density of $n_{\mathrm{H}}=3 \times 10^{3} \mathrm{~cm}^{-3}$ while the visual extinction has reached $A_{V}=5$. The only significant way to deplete $\mathrm{CO}$ from the gas phase beyond this point is through $\mathrm{CO}$ freeze-out on grain surfaces. We can see this happening by the decrease in $\mathrm{CO}$ abundance after $t=1.65 \times 10^{7} \mathrm{yr}$. A full layer of water ice has covered the dust surface at this point. See Fig. 5 bottom panel where the black (water) line crosses the dotted line. The CO abundance drops to $\sim 2 \times 10^{-5}$ near the end of our simulation. We perceive that it continues to drop to $\sim 2 \times 10^{-6}$ at $t=2.2 \times 10^{7} \mathrm{yr}$, which extends beyond the plot range. Oxygen is also increasingly depleted from the gas phase when the dust is enveloped by a mantle of water ice. Neutral oxygen has a steeper decline than $\mathrm{CO}$, with an abundance of $n_{\mathrm{O}} / n_{\mathrm{H}}=1.5 \times 10^{-6}$ at $t=2 \times 10^{7} \mathrm{yr}$. This means that oxygen is also being locked up in something other than $\mathrm{CO}$, which we know to be mostly water ice.

Within our densest clump, we never reach a situation with a $\mathrm{C} / \mathrm{O}$ abundance ratio of unity. A $\mathrm{C} / \mathrm{O}$ ratio above unity might lead 

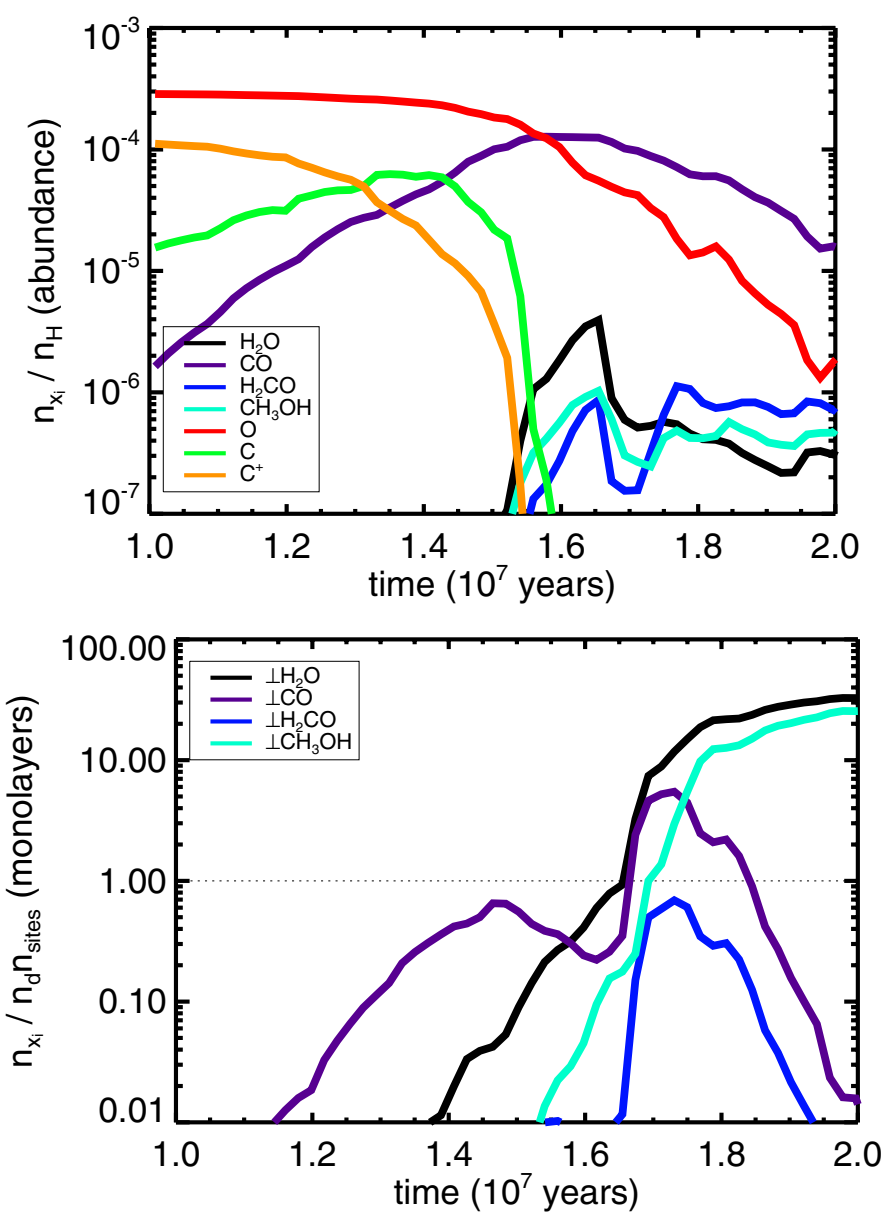

Fig. 5. Species abundances varying with time. The top panel displays the abundance of gas-phase species as a function of time. The bottom panel shows the growth of ice layers on grain surfaces. Each species is represented by a different color.

to interesting carbon chemistry in the gas phase and is attained by models using varying local interstellar background radiation fields, $G_{0}=1-1000$ (Hollenbach et al. 2009). We remind that we use $G_{0}=1$. The ratio we obtain always remains below unity and consistently below 0.01 above $t=1.55 \times 10^{7} \mathrm{yr}$, when more than half of the dust surface is covered.

The gas-phase abundances of water (black), methanol (light blue), and formaldehyde (dark blue) rise above a value of $10^{-7}$ after 15 Myr (Fig. 5), but remain relatively constant with abundances that, in the same order, linger around 3.2, 4.7, $7.5 \times 10^{-7}$ at the end of our simulation. The $\mathrm{H}_{2} \mathrm{CO} / \mathrm{CH}_{3} \mathrm{OH}$ ratio at that moment is 1.6 , which is a consequence of the high chemical desorption of formaldehyde. The abundances of species inside the clump after 20 Myr of cloud evolution are listed in Table 3.

We reach a high amount of methanol ice at the end of our simulation. This is partially because we did not incorporate species larger than methanol in our network. In addition to this, we have not considered diffusion through tunneling for oxygen atoms (Minissale et al. 2013a), which should lead to a lower methanol and a higher $\mathrm{CO}_{2}$ abundance.

\section{Ice species abundances}

The lower panel of Fig. 5 displays the abundance of frozen species in terms of ice layers covering the surface of the dust, which are given in units of monolayers along the $Y$-axis. Dust grains within the clump grow thicker ice mantles as a function
Table 3. Abundances of species at the end of the simulation $\left(n_{x_{i}} / n_{\mathrm{H}}\right)$.

\begin{tabular}{lclc}
\hline \hline Species & Final abundance & Species & Final abundance \\
\hline $\mathrm{H}$ & $2.1 \times 10^{-4}$ & $\mathrm{HCO}^{+}$ & $8.4 \times 10^{-11}$ \\
$\mathrm{H}^{-}$ & $9.4 \times 10^{-13}$ & $\mathrm{H}_{2} \mathrm{CO}$ & $7.5 \times 10^{-7}$ \\
$\mathrm{H}^{+}$ & $7.7 \times 10^{-10}$ & $\mathrm{CH}_{3} \mathrm{O}$ & $<10^{-20}$ \\
$\mathrm{H}_{2}$ & 0.49 & $\mathrm{CH}_{3} \mathrm{OH}$ & $4.7 \times 10^{-7}$ \\
$\mathrm{H}_{2}^{+}$ & $6.5 \times 10^{-11}$ & $\mathrm{e}^{-}$ & $6.2 \times 10^{-7}$ \\
$\mathrm{H}_{3}^{+}$ & $2.9 \times 10^{-9}$ & $\perp \mathrm{H}$ & $2.0 \times 10^{-10}$ \\
$\mathrm{O}^{-}$ & $1.5 \times 10^{-6}$ & $\perp \mathrm{H}_{\mathrm{c}}$ & $2.2 \times 10^{-6}$ \\
$\mathrm{O}^{-}$ & $1.2 \times 10^{-16}$ & $\perp \mathrm{H}_{2}$ & $1.1 \times 10^{-6}$ \\
$\mathrm{O}^{+}$ & $6.8 \times 10^{-16}$ & $\perp \mathrm{O}$ & $1.1 \times 10^{-12}$ \\
$\mathrm{O}_{2}$ & $3.4 \times 10^{-6}$ & $\perp \mathrm{O}_{2}$ & $2.1 \times 10^{-10}$ \\
$\mathrm{C}^{-}$ & $2.6 \times 10^{-10}$ & $\perp \mathrm{O}_{3}$ & $5.8 \times 10^{-13}$ \\
$\mathrm{C}^{-}$ & $1.1 \times 10^{-16}$ & $\perp \mathrm{OH}^{-12}$ & $2.8 \times 10^{-12}$ \\
$\mathrm{C}^{+}$ & $1.7 \times 10^{-13}$ & $\perp \mathrm{CO}^{-7}$ & $6.2 \times 10^{-8}$ \\
$\mathrm{OH}^{+}$ & $3.0 \times 10^{-7}$ & $\perp \mathrm{CO}_{2}$ & $2.4 \times 10^{-8}$ \\
$\mathrm{OH}^{+}$ & $1.1 \times 10^{-12}$ & $\perp \mathrm{H}_{2} \mathrm{O}$ & $1.5 \times 10^{-4}$ \\
$\mathrm{CO}_{\mathrm{CO}_{2}}$ & $1.5 \times 10^{-5}$ & $\perp \mathrm{HO}_{2}$ & $2.5 \times 10^{-12}$ \\
$\mathrm{H}_{2} \mathrm{O}$ & $5.1 \times 10^{-9}$ & $\perp \mathrm{H}_{2} \mathrm{O}_{2}$ & $5.7 \times 10^{-13}$ \\
$\mathrm{H}_{2} \mathrm{O}^{+}$ & $3.2 \times 10^{-7}$ & $\perp \mathrm{HCO}^{+}$ & $8.6 \times 10^{-12}$ \\
$\mathrm{H}_{3} \mathrm{O}^{+}$ & $3.3 \times 10^{-12}$ & $\perp \mathrm{H}_{2} \mathrm{CO}$ & $8.5 \times 10^{-9}$ \\
$\mathrm{HCO}$ & $8.5 \times 10^{-11}$ & $\perp \mathrm{CH}_{3} \mathrm{O}$ & $7.7 \times 10^{-12}$ \\
\hline
\end{tabular}

Notes. The symbol $\perp$ denotes a bound/ice species. $\perp \mathrm{H}_{\mathrm{c}}$ is the chemically adsorbed counterpart of $\perp \mathrm{H}$.

of time, which starts to level off around $t=1.8 \times 10^{7} \mathrm{yr}$. The maximum number of ice layers is mainly limited by the total surface area and the availability of the oxygen atoms, $n_{\mathrm{O}} / n_{\mathrm{H}}=$ $2.9 \times 10^{-4}$ (Table 1 ). We reach a total of 59 ice layers at the end of our simulation. This means that eventually $97 \%$ of the oxygen freezes out on dust and that only $3 \%$ resides in the gas phase locked in CO. The majority of ices are in the form of $\perp \mathrm{H}_{2} \mathrm{O}$, $\perp \mathrm{CO}, \perp \mathrm{H}_{2} \mathrm{CO}$ (formaldehyde), or $\perp \mathrm{CH}_{3} \mathrm{OH}$ (methanol).

When the cloud is still in a diffuse stage early on in cloud evolution, $\perp \mathrm{CO}$ is the predominant surface-bound species, with frozen water only second to $\perp C O$. This means that the initial ice mantle is well mixed. This contradicts the idea that the first ice layer should consist of water ice alone. The $\perp \mathrm{CO} / \perp \mathrm{H}_{2} \mathrm{O}$ ratio is about unity when half the dust surface is covered. This ratio decreases quickly over time due to the repeated hydrogenation of $\perp \mathrm{CO}$ to eventually form formaldehyde and methanol. The amount of $\perp \mathrm{CO}$ steeply decreases after the first ice layer has formed, while water ice grows more rapidly with increasing density. $\mathrm{CO}$ and water each dominate the surface coverage at different epochs, but water ice always dominates the ice composition when more than one layer of ice covers the dust. The dominance of solid $\mathrm{CO}$ on surfaces lasts for about $3 \mathrm{Myr}$ in our simulation, which is only a fraction of a cloud dynamical time, whereafter water ice becomes the dominant ice species. We expect that frozen $\mathrm{CO}$ can only be observed mixed with ices.

As stated earlier, the first mly of ice is formed at $t=1.65 \times$ $10^{7} \mathrm{yr}$ after we start our simulation. More importantly than time, the first ice layer is formed when the cloud is dense and cold enough, at which point it enters a molecular stage. Before that, we find that solid species start to grow rapidly, that is, beyond $0.1 \mathrm{mly}$, above a density of $n_{\mathrm{H}}=360 \mathrm{~cm}^{-3}$, with $n_{\mathrm{H}_{2}} / \mathrm{n}_{\mathrm{HI}}=2$, and below a gas temperature of $T_{\mathrm{g}}=16 \mathrm{~K}$. The cloud environment where these frozen species form has an $A_{V} \geq 1.8$ with a dust temperature of $7.5 \mathrm{~K}$. Water ice becomes the main constituent of the icy surface when the gas density rises above $n_{\mathrm{H}}=2.0 \times 10^{3}$ and the gas temperature falls below $11.5 \mathrm{~K}$. The visual extinction 

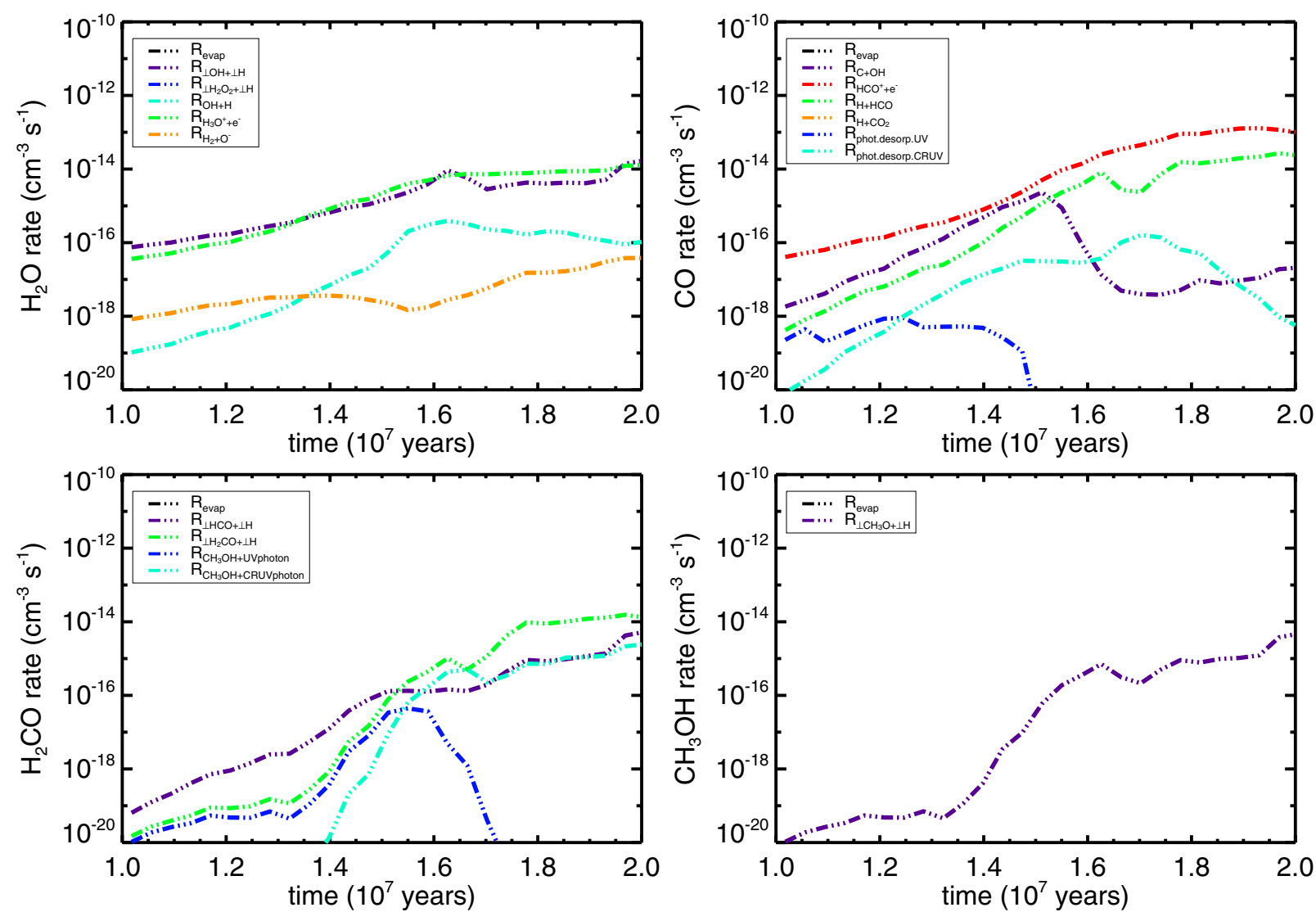

Fig. 6. Formation rates of gas-phase species. The main formation rates of $\mathrm{H}_{2} \mathrm{O}$ (top left), $\mathrm{CO}$ (top right), $\mathrm{H}_{2} \mathrm{CO}$ (bottom left), and $\mathrm{CH}_{3} \mathrm{OH}($ bottom right) are plotted as a function of time in $10^{7} \mathrm{yrs}$. $R_{\text {evap }}$, the evaportation rate, is drawn for each specie, however, this rate is quite low for methanol such that the line does not fall within the limits of the plot. Other molecular reactions as well as photo-processes that result in the four mentioned species are given within the legend.

of our clump now reaches upto 5.2. This also marks the turnover point where gas-phase CO starts to become depleted and the gas and dust temperatures start rising. More than two-thirds of the dust surface is covered by ice at this time. This in turn shifts the adsorption energies of the species from bare surface to icy surface binding energies, see Table 2 . In most cases, the binding energies increase when the substrate becomes water ice. These changes affect the thermal evaporation and the reaction rates on dust surfaces, as can be seen by Eqs. (10) and (12). Where the former one causes a build-up of ices by inhibiting evaporation, the latter slows down the formation of species by reducing the mobility. Moreover, the chemical desorption probabilities also drop by a factor of 5 when the dust is covered by ice, adding to the ice build-up. The combined effects work in favor of constructing more ices. This is shown by the sharp rise in the curves in Fig. 5 around the point where one mly of ice has formed.

\subsection{Time evolution of species formation rates}

By following the formation rates of the species in our model, we can understand the preferred formation pathways for each of our species. Here we report the formation rates of the species $\mathrm{H}_{2} \mathrm{O}, \mathrm{CO}, \mathrm{H}_{2} \mathrm{CO}$, and $\mathrm{CH}_{3} \mathrm{OH}$ during collapse. In the following images, Figs. 6 (gas species) and 7 (ice species), we plot the main formation rates for each of these species.

\section{Gas-phase formation rates}

In the top left panel of Fig. 6, we see that gas-phase water has two main formation channels. One through the reaction of
$\mathrm{H}_{3} \mathrm{O}^{+}$with $\mathrm{e}^{-}$in the gas phase, the other through chemical desorption by the reaction of $\perp \mathrm{OH}$ with $\perp \mathrm{H}$ on dust surfaces from which $90 \%$ of the reactions on bare grain surfaces desorb into the gas phase. This drops to $18 \%$ when the surface is covered by ice. This means that if the dust formation channel is not considered, the gas-phase water formation rate will typically be underestimated by a factor of $\sim 2$ with the conditions used in our simulations. Other channels, such as $\mathrm{OH}$ with $\mathrm{H}, \mathrm{H}_{2}$ with $\mathrm{O}^{-}$, and the second important desorption channel $\perp \mathrm{H}_{2} \mathrm{O}_{2}$ with $\perp \mathrm{H}$ are less significant routes to form gas-phase water. The latter channel may become much more relevant if reactiondiffusion competition is considered for tunneling because of the $\operatorname{high}\left(E_{\mathrm{a}}=1000 \mathrm{~K}\right)$ barrier.

In the top right panel of Fig. 6, we address the formation rates of $\mathrm{CO}$ in the gas phase. $\mathrm{CO}$ mainly forms through the dissociative recombination reaction $\mathrm{HCO}^{+}+\mathrm{e}^{-}$. This recombination rate is quite high as long as there are enough electrons around. The $\mathrm{CO}$ formation rate is also strongly dependent on the HCO supply in the gas phase, in which, as we show below, surface chemistry plays a major role. Below $n_{\mathrm{H}}=10^{3} \mathrm{~cm}^{-3}, \mathrm{HCO}^{+}$is primarily formed by the reaction $\mathrm{C}^{+}$with $\mathrm{H}_{2} \mathrm{O}$, but at higher densities $\mathrm{HCO}^{+}$is mainly produced through the ionization of chemically desorbed HCO. The second important formation route to form $\mathrm{CO}$ is through $\mathrm{C}+\mathrm{OH}$, but only early on in cloud evolution, that is, when the cloud is still in a diffuse stage. The CO formation rate by $\mathrm{C}+\mathrm{OH}$ sharply decreases after it peaks around $t=1.5 \times 10^{7} \mathrm{yr}$. This is because atomic carbon is becoming depleted as more and more carbon is converted into $\mathrm{CO}$ and $\mathrm{HCO}$. Once atomic carbon is depleted, the remainder of $\mathrm{O}$ and $\mathrm{OH}$ can 
S. Hocuk and S. Cazaux: Interplay of gas and ice during cloud evolution
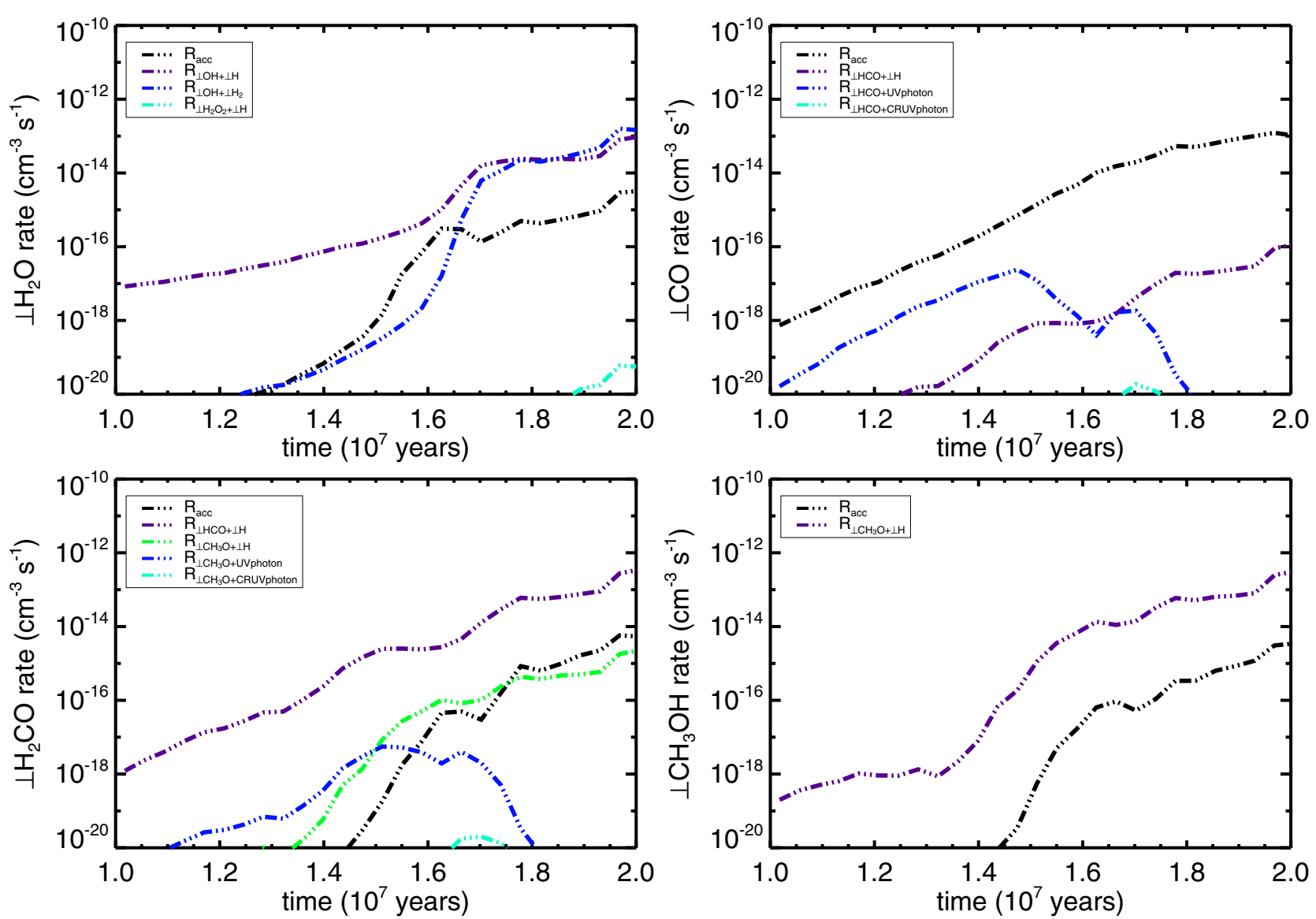

Fig. 7. Formation rates of ice species. The main formation rates of $\perp \mathrm{H}_{2} \mathrm{O}$ (top left), $\perp \mathrm{CO}$ (top right), $\perp \mathrm{H}_{2} \mathrm{CO}$ (bottom left), and $\perp \mathrm{CH} \mathrm{H}_{3} \mathrm{OH}($ bottom right) are plotted as a function of time in $10^{7} \mathrm{yr}$. Surface reactions and photo-processes that result in the four mentioned species are given within the legend.

be channeled into other reactions. We can see, for example, an upturn in the water formation rate through the reaction of $\mathrm{OH}+\mathrm{H}$ at the same moment in time. $\mathrm{H}+\mathrm{HCO}$ becomes the second-most important channel at later stages $\left(t>1.55 \times 10^{7} \mathrm{yr}\right)$ as we enter the translucent cloud stage $\left(n_{\mathrm{H}}>10^{3} \mathrm{~cm}^{-3}\right)$. Even though these are all gas-phase reactions, they are heavily affected by the production of $\mathrm{HCO}$ and $\mathrm{OH}$ on grain surfaces that are subsequently being released into the gas phase. Without the catalyzed species formation on dust, gas-phase $\mathrm{CO}$ formation rates will be constrained. We note that since hydrocarbon chemistry is beyond the scope of this work, an important $\mathrm{CO}$ formation channel in diffuse regions and at edges of molecular clouds, i.e., $\mathrm{CH}_{2}+\mathrm{O}$, is omitted (Tielens \& Hollenbach 1985; Keto \& Caselli 2008). Long carbon chain species, such as $\mathrm{C}_{9} \mathrm{H}^{+}$, which do not take part here, may also influence $\mathrm{CO}$ formation rates (Ruffle et al. 2002).

After the formation of the first ice layer at $t=1.65 \times 10^{7} \mathrm{yr}$, $\mathrm{H}$ and $\mathrm{HCO}$ are more strongly bound to the surface, see Table 2 for adsorption energies. This reduces the mobility of atoms on the dust surface as well as the desorption probabilities because they both depend on the binding energy, which explains the acute momentary decline in the second-most important rate at this time, $R_{\mathrm{H}+\mathrm{HCO}}$. We also see that photodesorption is not an important $\mathrm{CO}$ producer during the whole evolution of the cloud, from diffuse conditions to the first core formation. Even without our two-ice-layer penetration restriction for the UV photons, the photodesorption rates would still be an order of magnitude lower than the main $\mathrm{CO}$ formation rate. Since $\mathrm{CO}$ photodesorption occurs most efficiently by photons with energies of around $8-9 \mathrm{eV}$ (Muñoz Caro et al. 2010; Fayolle et al. 2011), while CO is photodissociated in the gas phase by UV photons with energies of $>11 \mathrm{eV}$, we might be underestimating the photodesorption rate when taking into account $\mathrm{CO}$ self-shielding for photodesorption. A test run without any self-shielding showed that the photodesorption rate is then much higher, and almost equal to the rate $R_{\mathrm{C}+\mathrm{OH}}$, but is still lower by more than one order of magnitude than the main $\mathrm{CO}$ formation rate $R_{\mathrm{HCO}^{+}+\mathrm{e}^{-}}$. This did not affect our results. Lastly, we can see that gaseous CO is not enhanced by thermal evaporation as the dust temperature is too cold $T_{\text {dust }}=7-8 \mathrm{~K}$ for $\perp \mathrm{CO}$ to be released. In fact, this will eventually lead to the depletion of $\mathrm{CO}$ from the gas phase as accretion starts to become more important with increasing density.

In the bottom left panel of Fig. 6, we display the formation rates of formaldehyde in the gas phase. The most dominant pathway to form formaldehyde in diffuse clouds $\left(t<1.5 \times 10^{7} \mathrm{yr}\right.$, $n_{\mathrm{H}}<10^{3} \mathrm{~cm}^{-3}$ ) is by direct chemical desorption of $\perp \mathrm{H}_{2} \mathrm{CO}$ following the reaction $\perp \mathrm{HCO}+\perp \mathrm{H}$. Only $1 \%$ (ice) to $5 \%$ (bare) of this reaction results in the formation of $\mathrm{H}_{2} \mathrm{CO}$ (gas), but it still emerges as the dominant rate. Guzmán et al. (2013) also concluded that the gas-phase formaldehyde formation is due to nonthermal desorption, but attributed its production to photodesorption. Van der Wiel et al. (2009) affirmed the need of a continuous supply of formaldehyde, for instance, from grain surface chemistry, to explain the abundances in their observations of the Orion Bar. Above $t=1.55 \times 10^{7} \mathrm{yr}$, chemical desorption following the reaction $\perp \mathrm{H}_{2} \mathrm{CO}+\perp \mathrm{H}$, together with the photodissociation of methanol in the gas by CRUV photons, overtakes the former $\left(R_{\perp \mathrm{HCO}+\perp \mathrm{H}}\right)$ desorption rate. In $10 \%$ (ice) to $50 \%$ (bare) of the cases, the reaction $\perp \mathrm{H}_{2} \mathrm{CO}$ with $\perp \mathrm{H}$ results in the desorption 
of the products. This reaction leads to the formation of a $\mathrm{CH}_{3} \mathrm{O}$ radical that may be released into the gas only to quickly form $\mathrm{H}_{2} \mathrm{CO}+\mathrm{H}$. The in-between steps are omitted here, since they are relatively fast. Thermal desorption of $\perp \mathrm{H}_{2} \mathrm{CO}$ is not a significant gas-phase supplier for formaldehyde either.

In the bottom right panel of Fig. 6, we show that methanol has only one essential formation mechanism. This is by chemical desorption from the grain surface reaction of $\perp \mathrm{CH}_{3} \mathrm{O}+\perp \mathrm{H}$. The rate remains below $10^{-14} \mathrm{~cm}^{-3} \mathrm{~s}^{-1}$, which means that it will not result in much methanol into the gas phase in a cloud lifetime of $\sim 10^{14} \mathrm{~s}$. Most of the methanol will remain frozen onto grain surfaces. The gas can only be enriched by methanol through evaporation of the frozen species at higher temperatures inside the cloud.

\section{Ice species formation rates}

In the top left panel of Fig. 7, we present the formation rates of water on surfaces. Water ice primarily forms by the reaction $\perp \mathrm{OH}+\perp \mathrm{H}$. This exothermic reaction occurs without a barrier and has a high chance to chemically desorb. The percentage that remains on the dust surface is $10 \%$, which increases to $82 \%$ for an icy substrate. This effect is directly visible in the figure from the jump in reaction rates at $t=1.65 \times 10^{7} \mathrm{yr}$ when the dust is covered by an ice mantle. The reaction $\perp \mathrm{OH}+\perp \mathrm{H}_{2}$ also increases greatly upon first ice layer formation. With $100 \%$ of the products remaining on dust, this reaction has a barrier of $E_{\mathrm{a}}=2100 \mathrm{~K}$ that needs to be overcome. Despite this, it dominates the water-ice formation rate at later times when the cloud density is higher, that is, $n_{\mathrm{H}} \geq 10^{4} \mathrm{~cm}^{-3}$ at the dense molecular stage. This is mainly due to the increasing $n_{\mathrm{H}_{2}} / n_{\mathrm{HI}}$ ratio during cloud evolution. Accretion of gaseous water becomes important at densities of $n_{\mathrm{H}} \simeq 4 \times 10^{3} \mathrm{~cm}^{-3}$, with gas and dust temperatures of around $10 \mathrm{~K}$. By forming $\mathrm{H}_{2} \mathrm{O}$ on dust, releasing it into the gas and reaccreting it back onto the dust will make the ice more porous. After fully covering the dust surface with water ice, the accretion rate decreases like the gas-phase water formation did as a result of the decline in the desorption probability of the main formation rate.

In the top right panel of Fig. 7, we give the formation rates of $\perp \mathrm{CO}$. CO ice grows on grain surfaces predominantly by accretion. The high $\mathrm{CO}$ abundance in the gas phase and the low gas and dust temperatures that allow for a high sticking coefficient result in a high accretion rate. The $\mathrm{CO}$ ice formation mechanism becomes somewhat circular above $t=15 \mathrm{Myr}$, because $\mathrm{CO}$ on grain surfaces are hydrogenated to form $\mathrm{HCO}$, thereupon to be chemically desorbed into the gas phase. The desorbed HCO molecule is quickly ionized through photoionization or ion exchange reactions to form $\mathrm{HCO}^{+}$, making it the primary route to form $\mathrm{HCO}^{+}$. This ion, as we know, dissociates into $\mathrm{CO}$ in the gas phase only to be reaccreted on dust grains where the whole cycle restarts. A demonstration of the cycle is given below.

$$
\mathrm{CO}_{\text {gas }} \stackrel{\mathrm{a}}{\rightarrow} \mathrm{CO}_{\text {ice }} \stackrel{\mathrm{b}}{\rightarrow} \mathrm{HCO}_{\text {ice }} \stackrel{\text { c }}{\rightarrow} \mathrm{HCO}_{\text {gas }} \stackrel{\mathrm{d}}{\rightarrow} \mathrm{HCO}_{\text {gas }}^{+} \stackrel{\mathrm{e}}{\rightarrow} \mathrm{CO}_{\text {gas }}
$$

$\mathrm{a}=$ accretion

$\mathrm{b}=$ hydrogenation

$\mathrm{c}=$ chemical desorption

$\mathrm{d}=$ ionization

$\mathrm{e}=$ dissociative recombination .

The chicken-and-egg problem is circumvented since CO initially forms in the gas phase through a channel independent of itself, namely $\mathrm{H}_{2} \mathrm{O}+\mathrm{C}^{+} \rightarrow \mathrm{HCO}^{+}+\mathrm{H}$ at $n_{\mathrm{H}}<10^{3} \mathrm{~cm}^{-3}$. This route is still associated with surface chemistry to a certain degree because gas-phase water formation is enhanced by the reactions on dust grains. A relatively high $\mathrm{CO}$ abundance is sustained in the gas phase by the continued supply through the cycle at low, $T_{\mathrm{d}}<10 \mathrm{~K}$, temperatures, which would otherwise result in the rapid freeze-out of $\mathrm{CO}$. Photodissociation by UV photons at low extinction $A_{V}<3$, and the dissociative reaction $\perp \mathrm{HCO}+\perp \mathrm{H}$ at high extinction $A_{V}>5$ are other, but minor producers of $\perp \mathrm{CO}$. Since the CO freeze-out occurs throughout cloud evolution, it is expected that $\mathrm{CO}$ ice will be present and well mixed within every ice layer covering the grain surfaces. The $\mathrm{CO}$ in the upper layers will subsequently become more and more hydrogenated as the medium density rises. This will decrease the amount of $\mathrm{CO}$ ice, but the first ice layer(s) should still have pristine $\mathrm{CO}$ ice mixed with water ice.

In the bottom left panel of Fig. 7, we report the rates needed to form $\perp \mathrm{H}_{2} \mathrm{CO}$. Formaldehyde ice mainly forms after two successive hydrogenations of $\perp \mathrm{CO}$. The reaction $\perp \mathrm{HCO}+\perp \mathrm{H}$ is, therefore, the main producer of formaldehyde ice throughout cloud evolution. This reaction has a $95 \%$ (bare) to $99 \%$ (ice) probability to let the product remain on the surface of the dust grains. Reaccretion of formaldehyde by the chemically desorbed $\perp \mathrm{H}_{2} \mathrm{CO}$ from the this reaction, as this is one of the main gas-phase suppliers, is low because of the low desorption percentages. De-hydrogenation (removing an $\mathrm{H}$ atom) of $\perp \mathrm{CH}_{3} \mathrm{O}$ also produces by about two orders of magnitude less formaldehyde ice than the main rate. This is because the reaction $\perp \mathrm{CH}_{3} \mathrm{O}+\perp \mathrm{H} \rightarrow \perp \mathrm{H}_{2} \mathrm{CO}+\mathrm{H}_{2}$ has a reaction barrier, albeit low, of $E_{\mathrm{a}}=150 \mathrm{~K}$ that needs to be overcome.

The bottom right panel of Fig. 7 shows the formation rates of $\mathrm{CH}_{3} \mathrm{OH}$ on surfaces. Methanol ice essentially forms by four repeated hydrogenations of $\mathrm{CO}$. In a similar fashion as formaldehyde, methanol ice forms through the exothermic reaction $\perp \mathrm{CH}_{3} \mathrm{O}+\perp \mathrm{H}$ with a $95 \%$ (bare) to $99 \%$ (ice) probability to make $\perp \mathrm{CH}_{3} \mathrm{OH}$. The formation rate is initially low because first $\perp \mathrm{CH}_{3} \mathrm{O}$ has to be created in ample amounts. The rate increases after $t=1.33 \times 10^{7} \mathrm{yr}$ when $\perp \mathrm{CH}_{3} \mathrm{O}$ is steadily formed, and this rate is similar to that of formaldehyde. Methanol will still be more abundant than formaldehyde since it is a more stable, strongly bound molecule. To break up methanol with a neutral hydrogen atom, a barrier of $E_{\mathrm{a}}=3000 \mathrm{~K}$ needs to be overcome, which allows it to survive and build up over time. Methanol is also being reaccreted by the molecules that were initially created on dust and were immediately desorbed into the gas.

A caveat in our chemical network is that we did not include species larger than methanol, nor did we treat all the associated ions of methanol in the gas phase. Even though the gasphase chemistry is not expected to be as important as grain surface chemistry, this will still create a sink out of methanol and therefore make us overestimate it, especially at later times.

\section{Destruction rates}

The most important destruction rates for the species discussed in this section are displayed in Fig. 8. For the gas phase, only the accretion rate of $\mathrm{CO}$ is presented in this figure to highlight the competition between the destruction rate of $\perp \mathrm{CO}$. Other accretion rates are shown in Fig. 7. For ice species, the dominant destruction rates are given with the addition of $\mathrm{CO}$ photodesorption and methanol dehydrogenation.

The $\mathrm{CO}$ that is accreted is quickly hydrogenated to form $\mathrm{HCO}$. At $t=16.5 \mathrm{Myr}$ the hydrogenation rate diminishes somewhat, which will result in the increase of $\perp \mathrm{CO}$. The accretion rate of $\mathrm{CO}$ also slows down shortly thereafter because the chemical desorption of $\mathrm{HCO}$ that influences the $\mathrm{CO}$ abundance in the gas phase is also reduced. In the end, a new balance is reached. Like $\mathrm{CO}$, formaldehyde ice is mainly destroyed by 


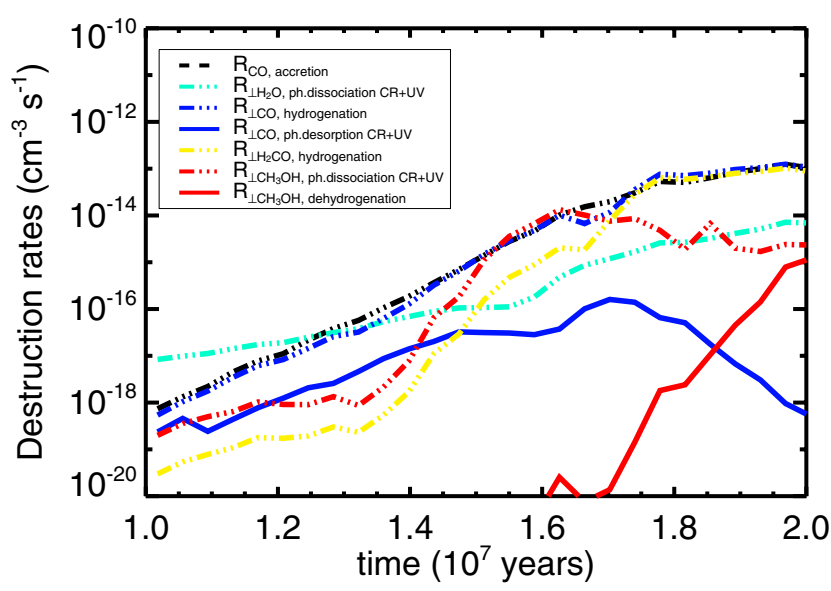

Fig. 8. Main destruction rates of key species. We plot the important destruction rates of $\mathrm{H}_{2} \mathrm{O}$ (ice), $\mathrm{CO}$ (gas+ice), $\mathrm{H}_{2} \mathrm{CO}$ (ice), and $\mathrm{CH}_{3} \mathrm{OH}$ (ice) as a funtion of time. The destruction of $\mathrm{CO}$ through surface accretion is drawn in black, $\perp \mathrm{CO}$ through hydrogenation and photodesorption in dark blue, $\perp \mathrm{H}_{2} \mathrm{O}$ through photodissociation in light blue, $\perp \mathrm{H}_{2} \mathrm{CO}$ through hydrogenation in yellow, and $\perp \mathrm{CH}_{3} \mathrm{OH}$ through photodissociation and dehydrogenation in red. For photo-processes, the rates obtained from cosmic rays and UV photons are added up.

hydrogenation. The rate is initially lower than that of $\mathrm{CO}$ due to the lower abundance of $\perp \mathrm{H}_{2} \mathrm{CO}$. That the two hydrogenation rates are equivalent at later times suggests that a balance is reached between formation and destruction. Methanol ice is mostly destroyed by radiation. Only at very late times, dehydrogenation of methanol becomes the strongest destruction mechanism. This is due to the high activation barrier $\left(E_{\mathrm{a}}=3000 \mathrm{~K}\right)$ in the dehydrogenation of methanol.

The main point is that all rates exhibit a twist in their curves at the time when ice completely covers the dust surface $(t=16.5 \mathrm{Myr})$. Following the transition in surface binding energies of species at this time, the reaction rates on grain surfaces change, that is, the mobility is reduced when the binding energies increase, while the chemical desorption probabilities decrease when the surface is covered by water ice.

\subsection{Ice distribution around the clump}

We also examined the abundance profiles of the ices within a radius of $5 \mathrm{pc}$ from the clump center. Inside this region, we followed the growth of ices on dust surfaces as a function of optical depth and present column density maps. We inspected the optical depth behavior for two different epochs, at a time of $t=16.6 \mathrm{Myr}$ and $t=18.2 \mathrm{Myr}$ after we started our simulation. In the period between these two time intervals, the transition from $\mathrm{CO}$ ice to methanol ice occurs inside the clump. The density of the clump center for the two snapshots is $n_{\mathrm{H}}=4 \times 10^{3} \mathrm{~cm}^{-3}$ and $10^{4} \mathrm{~cm}^{-3}$, respectively, while the gas temperature in both time frames lingers around $11 \mathrm{~K}$ (see Fig. 4). The gas temperature in the border region $\left(A_{V} \lesssim 1\right)$ is higher $T_{\mathrm{g}} \geq 20 \mathrm{~K}$, while the dust temperature is low $T_{\mathrm{d}} \sim 7 \mathrm{~K}$. Our resolution limits us in mapping the inner pc of our clump, and we did not have many data points at the center. This makes our curves appear rather smooth. A least-squares fifth-order polynomial was fitted to our data points and with this, the ice distributions for the two epochs are displayed in Fig. 9.

A thicker layer of ice covers the surface of the dust in the inner parts of the clump where $A_{V}$ and density are higher. Solid CO is more extended than solid water at a surface coverage below one mly. $\perp \mathrm{CO}$, however, also strongly contributes at the center of the clump. This establishes that water and $\mathrm{CO}$ ices are well mixed everywhere in the clump during the first ice layer formation. Clearly water ice becomes the main ice constituent of the icy mantle when one mly of water ice has formed. The transition at which solid water becomes more important than solid $\mathrm{CO}$ occurs at $A_{V}=4.8$ during the translucent cloud stage (see left panel of Fig. 9), while 1.6 Myr later, at a dense molecular stage, this transition takes place at $A_{V}=2.3$ (see right panel of Fig. 9). Deeper inside the core, methanol and formaldehyde ice is rapidly being formed at the loss of $\perp \mathrm{CO}$. The methanol ice surface coverage surpasses the $\mathrm{CO}$ ice coverage around an $A_{V}$ of 7 at $t=1.82 \times 10^{7} \mathrm{yr}$, but this transition also shifts to lower $A_{V}$ at later times. For example, the transition occurs at $A_{V}=4.5$ at $t=2 \times 10^{7} \mathrm{yr}$.

From the difference between two time frames, we can understand that the composition of the ice mantles do not strongly depend on the optical depth, since we have different compositions at different times for a given value of $A_{V}$. We can infer from this that photo-processes with a strong dependence on optical depth, such as photodissociation and photodesorption, are not significant factors to the ice composition within the conditions used in this work.

We also present the distribution of ices in spatial dimensions. In Fig. 10 we show maps of the species as they would appear on the sky. Here we plot the line-of-sight column densities of the species in and around the dense clump. These maps were created at the time of $t=1.82 \times 10^{7} \mathrm{yr}$ after simulation start, which is the same time as in the right panel of Fig. 9. The column densities range from $10^{10} \mathrm{~cm}^{-2}$ to $10^{20} \mathrm{~cm}^{-2}$ for all species. Water has the strongest peak at the center, followed by methanol. $\perp \mathrm{CO}$ has a weaker central feature, but is more extended. Most of the cells are colored green with columns of around $N_{\mathrm{CO}}=10^{16} \mathrm{~cm}^{-2}$. This tells us that $\mathrm{CO}$ ices will be present for a wide range of environmental conditions. Methanol, on the other hand, is very centralized with a high peak at the center, but with very little methanol ice extending outward. At this stage, water ice and methanol ice emission lines should be the easiest to detect; they have column densities of $10^{19} \mathrm{~cm}^{-2}$ and above inside the clump.

\section{Summary of conclusions and discussion}

We performed for the first time hydrodynamical simulations of a collapsing gas cloud with detailed gas and grain surface chemistry in which the interplay between gas and dust is interlinked with the thermodynamics of the cloud. We presented our results on the impact of dust chemistry on the formation of ices and the enrichment of gas-phase species during the evolution of a gas cloud. We also revealed the dominant formation routes for several species at different stages of cloud evolution.

To obtain our results, we followed a gas cloud from a fully diffuse atomic stage $\left(n_{\mathrm{H}}=10 \mathrm{~cm}^{-3}\right)$, which contracted and underwent phase transitions to finally form molecular clumps $\left(n_{\mathrm{H}}>10^{4} \mathrm{~cm}^{-3}\right)$. During the simulation, thick, $n_{\mathrm{mly}}=59$, ice layers formed within the densest clump. We find that the first ice layer covering the surface of the dust has a strong presence of adsorbed $\mathrm{CO}$. This mainly comes from accretion of $\mathrm{CO}$ from the gas phase, since gaseous $\mathrm{CO}$ is in ample supply. However, to form $\mathrm{CO}$ in the gas phase, the gas cloud needs to be supplied with $\mathrm{H}_{2} \mathrm{O}, \mathrm{HCO}$, and $\mathrm{OH}$ in the first place $\left(\mathrm{CH}_{2}\right.$ not included in this work). During the translucent and molecular cloud stages, these species are mostly formed on dust grains to be subsequently released into the gas phase through the process of 

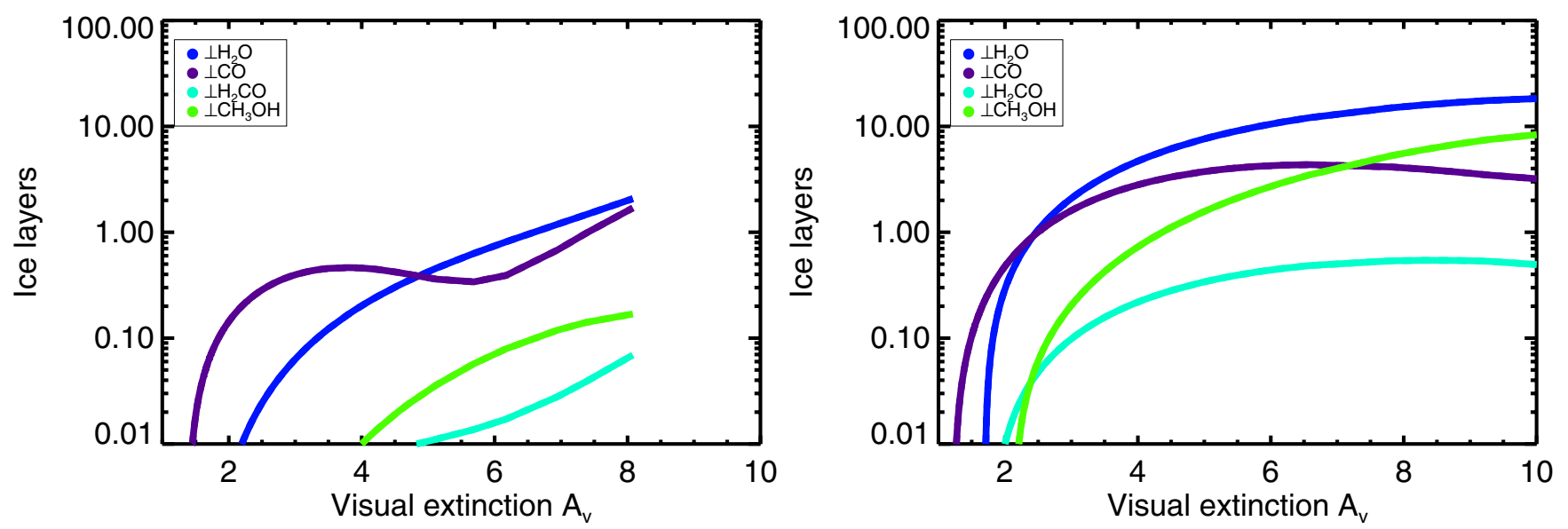

Fig. 9. Distribution of ices in and around a molecular clump. The amount of ice layers covering the surface of dust for several species is plotted as a function of visual extinction. The left panel displays the environment of a clump at a time of $t=1.66 \times 10^{7} \mathrm{yr}$ of cloud evolution. The right panel shows a time snapshot of the clump after $t=1.82 \times 10^{7} \mathrm{yr}$ of cloud evolution. The colored curves are least-squares fits to simulation data points.

chemical desorption. Without the enhanced HCO formation in translucent clouds through the exothermic reaction $\perp \mathrm{H}+\perp \mathrm{CO}$ at $n_{\mathrm{H}} \geq 10^{3} \mathrm{~cm}^{-3}$, CO in the gas phase would form at a much lower rate. We conclude from this that gas-phase $\mathrm{CO}$ formation requires grain surface reactions to be effective when starting from fully atomic conditions. We also conclude that in this chemical desorption is essential in supplying HCO to the gas, but note that the desorption rate is sensitive to the activation barrier of the reaction.

Our results show us that the first ice layer is formed during the translucent cloud stage at a density of $n_{\mathrm{H}}=4 \times 10^{3} \mathrm{~cm}^{-3}$. $\mathrm{CO}$ ice is well mixed at this stage with water ice. After one mly of ice has formed, freeze-out occurs more rapidly as a result of the change in binding energy of the species with respect to an icy surface. This influences the mobility of species on surfaces as well as the chemical desorption probabilities, which eventually causes the formation of more water ice. We also see that CO ice gradually decreases with increasing density and opacity because it increasingly is more hydrogenated through the successive reactions with $\perp \mathrm{H}$ to form formaldehyde and methanol.

From the distribution of ices in the region surrounding the clump we see that most of the ices are at the clump center. CO ice is more extended toward the outer regions than water ice, and it is consumed in the clump center to form formaldehyde and methanol. Methanol favors the high-density regions, where it becomes the second-most abundant ice after water ice.

We conclude that grain surface chemistry strongly affects the abundances of species in the gas phase by creating strong, sometimes dominant, pathways for forming key molecules, such as water, formaldehyde, and methanol. Gas-phase species are also depleted by freeze-out onto dust surfaces at different cloud evolutionary stages. Water and especially CO freeze-out is seen to occur, which is confirmed by rates. Dust chemistry also allowed us to follow the formation and build-up of ices during the evolution of a gas cloud. Water ice becomes the dominant constituent of the ice mantle after $15.5 \mathrm{Myr}$ of cloud evolution when the density has risen above $2 \times 10^{3} \mathrm{~cm}^{-3}$, and methanol ice has a strong $\sim 43 \%$ presence inside dense clumps. These chemical influences affect the thermodynamic properties of the progenitors of star-forming regions. Moreover, we note that these changes occur and have an impact at early, translucent cloud stages that evolve into molecular clouds. These are critical moments for cloud fragmentation.

In summary, our results are that

- in the first ice layer(s), $\mathrm{CO}$ ice is well mixed with $\mathrm{H}_{2} \mathrm{O}$ ice;

- freeze-out of species greatly increases after one mly of ice covers the dust surface;

- chemical desorption from grain surfaces can be important in supplying the gas phase with $\mathrm{CO}$ and formaldehyde;

- methanol and formaldehyde (gas) is seen in higher density regions $\left(n_{\mathrm{H}}>4 \times 10^{3} \mathrm{~cm}^{-3}\right)$, while formaldehyde ice and $\mathrm{CO}$ ice are more likely to be found in the surrounding area; and finally;

- surface chemistry alters the species abundances in translucent clouds, which will affect the whole cloud evolution.

\subsection{Discussion}

Our chemical network consists of 42 species and 257 reactions. Although this is a respectable amount given the complexity of this work, the network is still restricted. In this, methanol is the largest molecule that we allow to form in our network. This will inevitably create a sink out of this molecule and makes us overestimate the methanol abundance since there are no routes to form higher order molecules or ions.

Chemical desorption following the hydrogenation of $\mathrm{CO}$ and formaldehyde is sensitive to the activation barriers we used. The activation barriers for the reactions $\mathrm{CO}+\mathrm{H}$ and $\mathrm{H}_{2} \mathrm{CO}+\mathrm{H}$ are sometimes found to be high, 2000-2500 K (e.g., Garrod et al. 2008; Peters et al. 2013). Such high activation barriers would cause much less $\mathrm{CO}$ hydrogenation and chemical desorption to occur. Monte Carlo simulations often use these high activation barriers, but also naturally consider the reaction-diffusion competition in their kinematic models. The reaction-diffusion competition makes the reactions much more likely to occur. Because we did not incorporate the reaction-diffusion competition, we used an "effective" activation barrier to account for this process. We adopted "effective" barriers of $600 \mathrm{~K}$ for $\mathrm{CO}+\mathrm{H}$ and $400 \mathrm{~K}$ for $\mathrm{H}_{2} \mathrm{CO}+\mathrm{H}$ (Awad et al. 2005; Fuchs et al. 2009) instead of the high values considered for quantum tunneling when the reactiondiffusion competition is taken into account (5400-9600 K, Awad et al. 2005). However, recent experiments by Minissale et al. (priv. comm.) tend to find barriers on the order of $1000 \mathrm{~K}$. The 

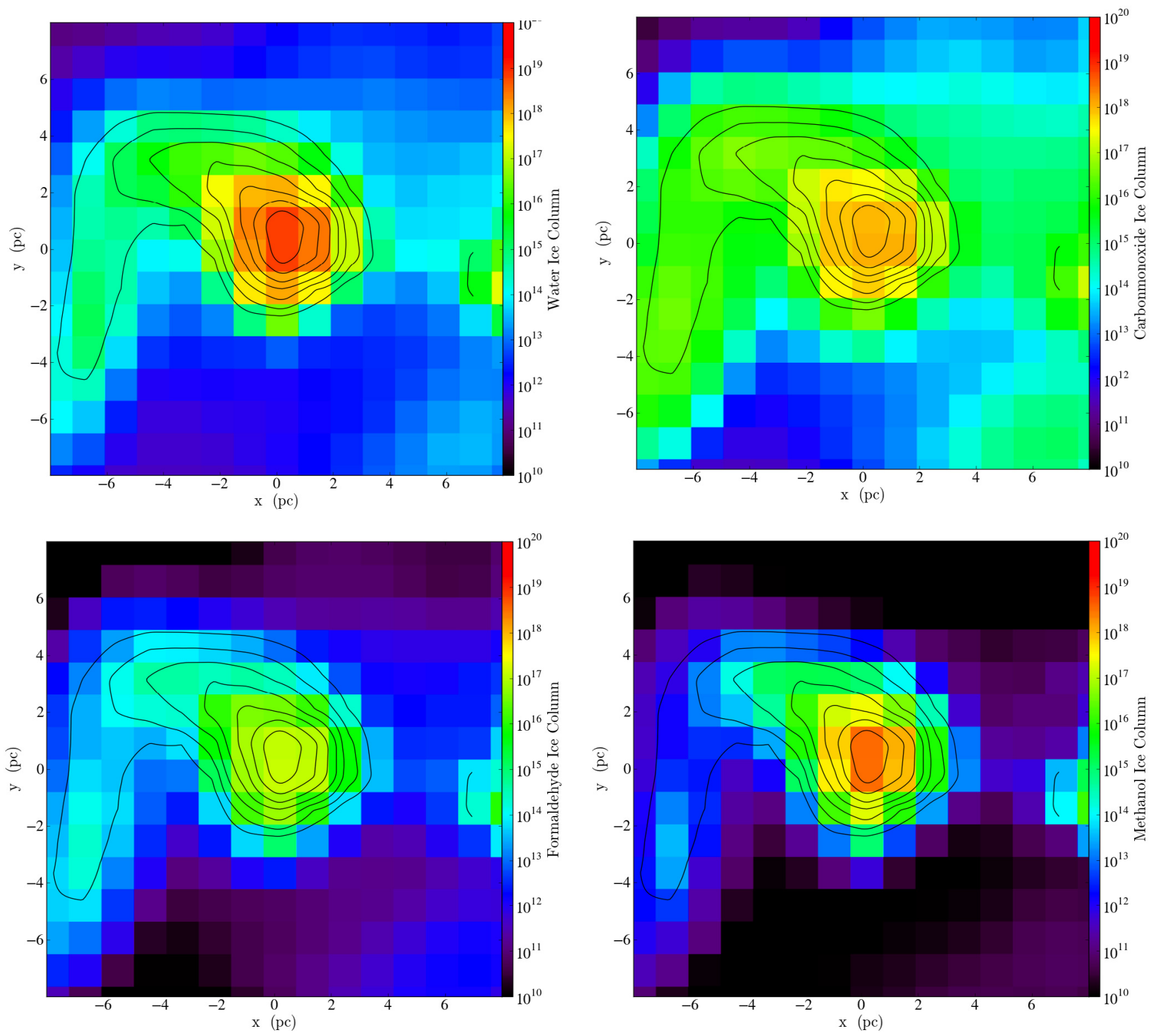

Fig. 10. Ice maps. In these images, a projection along the $Z$-axis, resulting in a line-of-sight column $\left(\mathrm{cm}^{-2}\right)$, is plotted within a $16 \times 16 \mathrm{pc}$ box. From top left to bottom right, ice maps of the species $\perp \mathrm{H}_{2} \mathrm{O}, \perp \mathrm{CO}, \perp \mathrm{H}_{2} \mathrm{CO}$, and $\perp \mathrm{CH}_{3} \mathrm{OH}$ are displayed. The color range representing the species column densities spans from low $10^{10}$ (black) $\mathrm{cm}^{-2}$ to high $10^{20} \mathrm{~cm}^{-2}$ (red) values. The contours enclose the region where the total column density, $N_{\mathrm{H}}=\int n_{\mathrm{H}} \mathrm{d} s$, ranges from $10^{22}$ to $6 \times 10^{22} \mathrm{~cm}^{-2}$, i.e., $A_{V}=4.5-27$, to indicate where most of the matter lies.

barriers we used in that case would overestimate the hydrogenation of CO. The exact content of the ices at long time scales will be re-addressed in a future work, once the barriers for the hydrogenation of $\mathrm{CO}$ and $\mathrm{H} 2 \mathrm{CO}$ are more clearly defined for the solid state.

Higher resolution simulations will allow us to probe the inner pc of a clump, which was not the prime target of this work. We resolved the Jeans length by 44 cells for the initial state, but our Jeans resolution drops to 1.2 cells at the final stages of our simulation (taking into account the increase in mean mololecular mass). This is lower by a factor three than the Truelove criterion (Truelove et al. 1997), but artificial fragmentation at this stage is not a concern, because our simulation concludes shortly after we drop below 4 cells. The Jeans resolution at the final stages of our simulation should not affect the results on which we focused, that is, the chemical reactions.

Higher resolution will also enable obtaining more clumps with a wider range of clump densities and opacities. A statistical study can be performed with such a set of clumps to determine wether our results hold true for the general case. We note that although the chemistry calculations are a local phenomenon, they reflect the cloud history and thus are affected by the evolution of the cloud, which makes the initial conditions (initial abundances, density profile, temperatures, background radiation field) and the large-scale physics (gravity, turbulence, feedback, magnetic fields) important for the results. However, because of its nature, grain surface chemistry acts as a balancing factor to the thermodynamics of the gas by the processes of freeze-out and by its catalytic nature, which causes it to form complex molecules. This, in the end, self-regulate the evolution of the cloud to some extent. To which degree this is true can be realized by a parameter study of simulations with a set of different initial and physical conditions.

A problem arising from our non-conserving numerical solver is that the ion-electron ratio is not conserved. We find that his occurs when the differential equation becomes stiff at higher, 
$n_{\mathrm{H}} \geq 4 \times 10^{3} \mathrm{~cm}^{-3}$, densities. The electron abundance diverges after 17 Myrs and is too high. This problem will have negligible effects on the thermal balance, while for the chemical evolution of the species other than electrons, we believe that the remaining simulation time is too short to have a strong impact.

Acknowledgements. This work is supported by the Netherlands Organization for Scientific Research (NWO). The authors thank P. Caselli, M. Spaans, M. Minissale, and F. Dulieu for all the useful discussions on various aspects of this work. The software used in this work was developed in part by the DOE NNSA ASC- and DOE Office of Science ASCR-supported Flash Center for Computational Science at the University of Chicago. The simulations have been run on the dedicated special purpose machines at the Kapteyn Astronomical Institute and at the Donald Smits Center for Information Technology using the Millipede Cluster, University of Groningen. Some of the kinetic data implemented in this work have been downloaded from the online database KiDA (Wakelam et al. 2012, http://kida.obs.u-bordeaux1.fr).

\section{References}

Agúndez, M., Goicoechea, J. R., Cernicharo, J., Faure, A., \& Roueff, E. 2010, ApJ, 713, 662

Andersson, S., \& van Dishoeck, E. F. 2008, A\&A, 491, 907

Andersson, S., Al-Halabi, A., Kroes, G.-J., \& van Dishoeck, E. F. 2006, J. Chem. Phys., 124, 064715

Anninos, P., Zhang, Y., Abel, T., \& Norman, M. L. 1997, Nature, 2, 209

Arab, H., Abergel, A., Habart, E., et al. 2012, A\&A, 541, A19

Arasa, C., Andersson, S., Cuppen, H. M., van Dishoeck, E. F., \& Kroes, G.-J. 2010, J. Chem. Phys., 132, 184510

Atkinson, R., Baulch, D. L., Cox, R. A., et al. 1989, J. Phys. Chem. Ref. Data, 18,881

Atkinson, R., Baulch, D. L., Cox, R. A., et al. 2004, Atmospheric Chemistry \& Physics, 4, 1461

Awad, Z., Chigai, T., Kimura, Y., Shalabiea, O. M., \& Yamamoto, T. 2005, ApJ, 626,262

Bahr, D. A., \& Baragiola, R. A. 2012, ApJ, 761, 36

Barzel, B., \& Biham, O. 2007, ApJ, 658, L37

Bec, J., \& Khanin, K. 2007, Phys. Rep., 447, 1

Bovino, S., Grassi, T., Latif, M. A., \& Schleicher, D. R. G. 2013, MNRAS, 434, L36

Burgers, J. M. 1939, Mathematical Examples Illustrating Relations Occurring in the Theory of Turbulent Fluid Motion, Kon. Ned. Akad. Wet., 17, 1

Cazaux, S., \& Tielens, A. G. G. M. 2004, ApJ, 604, 222

Cazaux, S., \& Tielens, A. G. G. M. 2010, ApJ, 715, 698

Cazaux, S., Tielens, A. G. G. M., Ceccarelli, C., et al. 2003, ApJ, 593, L51

Cazaux, S., Cobut, V., Marseille, M., Spaans, M., \& Caselli, P. 2010, A\&A, 522, A74

Chaparro Molano, G., \& Kamp, I. 2012, A\&A, 537, A138

Choi, Y., van der Tak, F., Bergin, E., \& Plume, R. 2013, in Protostars and Planets VI Posters, 7

Colella, P., \& Woodward, P. R. 1984, J. Comput. Phys., 54, 174

Collings, M. P., Dever, J. W., Fraser, H. J., \& McCoustra, M. R. S. 2003, Ap\&SS, 285,633

Cuppen, H. M., \& Herbst, E. 2007, ApJ, 668, 294

Dopcke, G., Glover, S. C. O., Clark, P. C., \& Klessen, R. S. 2011, ApJ, 729, L3

Draine, B. T. 1978, ApJS, 36, 595

Draine, B. T., \& Bertoldi, F. 1996, ApJ, 468, 269

Dubey, A., Antypas, K., Ganapathy, M. K., et al. 2009, Parallel Computing, 35, 512

Dulieu, F., Congiu, E., Noble, J., et al. 2013, Nature Sci. Rep., 3

Duncan, T. V., \& Miller, C. E. 2000, J. Chem. Phys., 113, 5138

Fayolle, E. C., Bertin, M., Romanzin, C., et al. 2011, ApJ, 739, L36

Fontani, F., Giannetti, A., Beltrán, M. T., et al. 2012, MNRAS, 423, 2342

Fryxell, B., Olson, K., Ricker, P., et al. 2000, ApJS, 131, 273

Fuchs, G. W., Cuppen, H. M., Ioppolo, S., et al. 2009, A\&A, 505, 629

Garrod, R. T., \& Pauly, T. 2011, ApJ, 735, 15

Garrod, R. T., Wakelam, V., \& Herbst, E. 2007, A\&A, 467, 1103

Garrod, R. T., Weaver, S. L. W., \& Herbst, E. 2008, ApJ, 682, 283

Gavilan, L., Lemaire, J. L., \& Vidali, G. 2012, MNRAS, 424, 2961

Goldsmith, P. F. 2001, ApJ, 557, 736

Güver, T., \& Özel, F. 2009, MNRAS, 400, 2050

Guzmán, V. V., Goicoechea, J. R., Pety, J., et al. 2013, A\&A, 560, A73

Habing, H. J. 1968, Bull. Astron. Inst. Netherlands, 19, 421

Heyer, M. H., \& Brunt, C. M. 2004, ApJ, 615, L45
Hocuk, S., \& Spaans, M. 2010, A\&A, 522, A24

Hocuk, S., \& Spaans, M. 2011, A\&A, 536, A41

Hocuk, S., Cazaux, S., \& Spaans, M. 2014, MNRAS, 438, L56

Hollenbach, D., \& McKee, C. F. 1979, ApJS, 41, 555

Hollenbach, D. J., Takahashi, T., \& Tielens, A. G. G. M. 1991, ApJ, 377, 192

Hollenbach, D., Kaufman, M. J., Bergin, E. A., \& Melnick, G. J. 2009, ApJ, 690, 1497

Indriolo, N., Geballe, T. R., Oka, T., \& McCall, B. J. 2007, ApJ, 671, 1736

Ioppolo, S., Sangiorgio, I., Baratta, G. A., \& Palumbo, M. E. 2013, A\&A, 554, A34

Jones, A. P., \& Williams, D. A. 1984, MNRAS, 209, 955

Karssemeijer, L. J., Ioppolo, S., van Hemert, M. C., et al. 2014, ApJ, 781, 16

Keto, E., \& Caselli, P. 2008, ApJ, 683, 238

Keto, E., Rawlings, J., \& Caselli, P. 2014, MNRAS, 440, 2616

Kristensen, L. E., van Dishoeck, E. F., van Kempen, T. A., et al. 2010, A\&A, 516, A57

Lamberts, T., Cuppen, H. M., Ioppolo, S., \& Linnartz, H. 2013, Phys. Chem. Chem. Phys., 15, 8287

Larson, R. B. 1981, MNRAS, 194, 809

Latif, M. A., Schleicher, D. R. G., \& Spaans, M. 2012, A\&A, 540, A101

Le Bourlot, J., Le Petit, F., Pinto, C., Roueff, E., \& Roy, F. 2012, A\&A, 541, A76

Lee, J. H., Michael, J. V., Payne, W. A., \& Stief, L. J. 1978, J. Chem. Phys., 69, 350

Lippok, N., Launhardt, R., Semenov, D., et al. 2013, A\&A, 560, A41

Liu, T., Wu, Y., \& Zhang, H. 2013, ApJ, 775, L2

Mathis, J. S., Rumpl, W., \& Nordsieck, K. H. 1977, ApJ, 217, 425

Meijerink, R., Cazaux, S., \& Spaans, M. 2012, A\&A, 537, A102

Miettinen, O., Harju, J., Haikala, L. K., \& Juvela, M. 2012, A\&A, 538, A137

Minissale, M., \& Dulieu, F. 2014, J. Chem. Phys., 141, 014304

Minissale, M., Congiu, E., Baouche, S., et al. 2013a, Phys. Rev. Lett., 111, 053201

Minissale, M., Congiu, E., Manicò, G., Pirronello, V., \& Dulieu, F. 2013b, A\&A, 559, A49

Miyauchi, N., Hidaka, H., Chigai, T., et al. 2008, Chem. Phys. Lett., 456, 27

Muñoz Caro, G. M., Jiménez-Escobar, A., Martín-Gago, J. Á., et al. 2010, A\&A, 522, A 108

Myers, P. C., \& Gammie, C. F. 1999, ApJ, 522, L141

Noble, J. A., Dulieu, F., Congiu, E., \& Fraser, H. J. 2011, ApJ, 735, 121

Noble, J. A., Congiu, E., Dulieu, F., \& Fraser, H. J. 2012a, MNRAS, 421, 768

Noble, J. A., Theule, P., Mispelaer, F., et al. 2012b, A\&A, 543, A5

Oba, Y., Watanabe, N., Hama, T., et al. 2012, ApJ, 749, 67

Öberg, K. I., van Dishoeck, E. F., \& Linnartz, H. 2009, A\&A, 496, 281

Omukai, K. 2000, ApJ, 534, 809

Peters, P. S., Duflot, D., Wiesenfeld, L., \& Toubin, C. 2013, J. Chem. Phys., 139, 164310

Plewa, T., \& Müller, E. 1999, A\&A, 342, 179

Pollack, J. B., Hollenbach, D., Beckwith, S., et al. 1994, ApJ, 421, 615

Roser, J. E., Vidali, G., Manicò, G., \& Pirronello, V. 2001, ApJ, 555, L61

Ruffle, D. P., Rae, J. G. L., Pilling, M. J., Hartquist, T. W., \& Herbst, E. 2002, A\&A, 381, L13

Sandford, S. A., \& Allamandola, L. J. 1988, Icarus, 76, 201

Schleicher, D. R. G., Galli, D., Palla, F., et al. 2008, A\&A, 490, 521

Spaans, M., \& Silk, J. 2000, ApJ, 538, 115

Stamatellos, D., Whitworth, A. P., Bisbas, T., \& Goodwin, S. 2007, A\&A, 475, 37

Suutarinen, A. N., Kristensen, L. E., Mottram, J. C., Fraser, H. J., \& van Dishoeck, E. F. 2014, MNRAS, 440, 1844

Tafalla, M., Santiago-García, J., Myers, P. C., et al. 2006, A\&A, 455, 577

Tielens, A. G. G. M., \& Hollenbach, D. 1985, ApJ, 291, 722

Truelove, J. K., Klein, R. I., McKee, C. F., et al. 1997, ApJ, 489, L179

Van der Wiel, M. H. D., van der Tak, F. F. S., Ossenkopf, V., et al. 2009, A\&A, 498, 161

van Dishoeck, E. F., Kristensen, L. E., Benz, A. O., et al. 2011, PASP, 123, 138

Visser, R., van Dishoeck, E. F., \& Black, J. H. 2009, A\&A, 503, 323

Wakelam, V., Herbst, E., Loison, J.-C., et al. 2012, ApJS, 199, 21

Walch, S. P., McMichael Rohlfing, C., Melius, C. F., \& Bauschlicher, Jr., C. W. 1988, J. Chem. Phys., 88, 6273

Weingartner, J. C., \& Draine, B. T. 2001, ApJ, 548, 296

Yamagishi, M., Kaneda, H., Ishihara, D., et al. 2013, ApJ, 773, L37

Yıldız, U. A., Acharyya, K., Goldsmith, P. F., et al. 2013, A\&A, 558, A58

Yu, H.-G., Muckerman, J. T., \& Sears, T. J. 2001, Chem. Phys. Lett., 349, 547

Yusef-Zadeh, F., Cotton, W., Viti, S., Wardle, M., \& Royster, M. 2013, ApJ, 764, L19

Zhen, J., \& Linnartz, H. 2014, MNRAS, 437, 3190 


\section{Appendix A: Reaction tables}

Here we list in tables the gas-phase and the surface reactions. Since adsorption reactions (Sect. 3.3.1) and thermal desorption reactions (Sect. 3.3.2) are relatively straightforward, they are omitted from this list. We also supply the adopted activation barriers, $E_{\mathrm{a}}$, in Kelvin within the relevant tables.

Table A.1. Photo-processes on surfaces.

\begin{tabular}{|c|c|c|}
\hline Reactants & & Products \\
\hline$\perp \mathrm{H}_{2}+\mathrm{CR}$ & $\rightarrow$ & $\perp \mathrm{H}+\perp \mathrm{H}$ \\
\hline$\perp \mathrm{O}_{2}+\mathrm{CRUV}$ & $\rightarrow$ & $\perp \mathrm{O}+\perp \mathrm{O}$ \\
\hline$\perp \mathrm{OH}+\mathrm{CRUV}$ & $\rightarrow$ & $\perp \mathrm{H}+\perp \mathrm{O}$ \\
\hline$\perp \mathrm{H}_{2} \mathrm{O}+\mathrm{CRUV}$ & $\rightarrow$ & $\perp \mathrm{H}+\perp \mathrm{OH}$ \\
\hline$\perp \mathrm{CO}_{2}+\mathrm{CRUV}$ & $\rightarrow$ & $\perp \mathrm{O}+\perp \mathrm{CO}$ \\
\hline$\perp \mathrm{HCO}+\mathrm{CRUV}$ & $\rightarrow$ & $\perp \mathrm{H}+\perp \mathrm{CO}$ \\
\hline$\perp \mathrm{H}_{2} \mathrm{CO}+\mathrm{CRUV}$ & $\rightarrow$ & $\perp \mathrm{H}+\perp \mathrm{HCO}$ \\
\hline$\perp \mathrm{CH}_{3} \mathrm{O}+\mathrm{CRUV}$ & $\rightarrow$ & $\perp \mathrm{H}+\perp \mathrm{H}_{2} \mathrm{CO}$ \\
\hline$\perp \mathrm{CH}_{3} \mathrm{OH}+\mathrm{CRUV}$ & $\rightarrow$ & $\perp \mathrm{H}+\perp \mathrm{CH}_{3} \mathrm{O}$ \\
\hline$\perp \mathrm{H}_{2}+$ UV Photon & $\rightarrow$ & $\perp \mathrm{H}+\perp \mathrm{H}$ \\
\hline$\perp \mathrm{O}_{2}+$ UV Photon & $\rightarrow$ & $\perp \mathrm{O}+\perp \mathrm{O}$ \\
\hline$\perp \mathrm{OH}+$ UV Photon & $\rightarrow$ & $\perp \mathrm{H}+\perp \mathrm{O}$ \\
\hline$\perp \mathrm{H}_{2} \mathrm{O}+$ UV Photon & $\rightarrow$ & $\perp \mathrm{H}+\perp \mathrm{OH}$ \\
\hline$\perp \mathrm{CO}_{2}+$ UV Photon & $\rightarrow$ & $\perp \mathrm{O}+\perp \mathrm{CO}$ \\
\hline$\perp \mathrm{HCO}+$ UV Photon & $\rightarrow$ & $\perp \mathrm{H}+\perp \mathrm{CO}$ \\
\hline$\perp \mathrm{H}_{2} \mathrm{CO}+$ UV Photon & $\rightarrow$ & $\perp \mathrm{H}+\perp \mathrm{HCO}$ \\
\hline$\perp \mathrm{CH}_{3} \mathrm{O}+\mathrm{UV}$ Photon & $\rightarrow$ & $\perp \mathrm{H}+\perp \mathrm{H}_{2} \mathrm{CO}$ \\
\hline$\perp \mathrm{CH}_{3} \mathrm{OH}+\mathrm{UV}$ Photon & $\rightarrow$ & $\perp \mathrm{H}+\perp \mathrm{CH}_{3} \mathrm{O}$ \\
\hline$\perp \mathrm{CO}+$ UV Photon & $\rightarrow$ & $\mathrm{CO}$ \\
\hline$\perp \mathrm{CO}+\mathrm{CRUV}$ & $\rightarrow$ & $\mathrm{CO}$ \\
\hline
\end{tabular}


Table A.2. Photo-processes in the gas phase.

\begin{tabular}{|c|c|c|c|c|c|c|c|c|}
\hline Reactants & & Products & Reactants & & Products & Reactants & & Products \\
\hline $\mathrm{H}+\mathrm{CR}$ & $\rightarrow$ & $\mathrm{H}^{+}+\mathrm{e}^{-}$ & $\mathrm{O}+\mathrm{CR}$ & $\rightarrow$ & $\mathrm{O}^{+}+\mathrm{e}^{-}$ & $\mathrm{CO}+\mathrm{CR}$ & $\rightarrow$ & $\mathrm{C}+\mathrm{O}$ \\
\hline $\mathrm{H}_{2}+\mathrm{CR}$ & $\rightarrow$ & $\mathrm{H}+\mathrm{H}$ & $\mathrm{H}_{2}+\mathrm{CR}$ & $\rightarrow$ & $\mathrm{H}+\mathrm{H}^{+}+\mathrm{e}^{-}$ & $\mathrm{H}_{2}+\mathrm{CR}$ & $\rightarrow$ & $\mathrm{H}^{+}+\mathrm{H}^{-}$ \\
\hline $\mathrm{H}_{2}+\mathrm{CR}$ & $\rightarrow$ & $\mathrm{H}_{2}^{+}+\mathrm{e}^{-}$ & $\mathrm{O}_{2}+\mathrm{CRUV}$ & $\rightarrow$ & $\mathrm{O}+\mathrm{O}$ & $\mathrm{OH}+\mathrm{CRUV}$ & $\rightarrow$ & $\mathrm{H}+\mathrm{O}$ \\
\hline $\mathrm{CO}_{2}+\mathrm{CRUV}$ & $\rightarrow$ & $\mathrm{O}+\mathrm{CO}$ & $\mathrm{H}_{2} \mathrm{O}+\mathrm{CRUV}$ & $\rightarrow$ & $\mathrm{H}+\mathrm{OH}$ & $\mathrm{HCO}+\mathrm{CRUV}$ & $\rightarrow$ & $\mathrm{H}+\mathrm{CO}$ \\
\hline $\mathrm{HCO}+\mathrm{CRUV}$ & $\rightarrow$ & $\mathrm{HCO}^{+}+\mathrm{e}^{-}$ & $\mathrm{H}_{2} \mathrm{CO}+\mathrm{CRUV}$ & $\rightarrow$ & $\mathrm{CO}+\mathrm{H}_{2}$ & $\mathrm{CH}_{4} \mathrm{OH}+\mathrm{CRUV}$ & $\rightarrow$ & $\mathrm{H}_{2}+\mathrm{H}_{2} \mathrm{CO}$ \\
\hline C + CRUV & $\rightarrow$ & $\mathrm{C}^{+}+\mathrm{e}^{-}$ & $\mathrm{CH}_{4} \mathrm{OH}+$ Photon & $\rightarrow$ & $\mathrm{H}_{2}+\mathrm{H}_{2} \mathrm{CO}$ & $\mathrm{H}_{2}^{+}+$Photon & $\rightarrow$ & $\mathrm{H}+\mathrm{H}^{+}$ \\
\hline $\mathrm{OH}^{+}+$Photon & $\rightarrow$ & $\mathrm{O}+\mathrm{H}^{+}$ & $\mathrm{H}_{3}^{+}+$Photon & $\rightarrow$ & $\mathrm{H}_{2}+\mathrm{H}^{+}$ & $\mathrm{H}_{3}{ }^{+}+$Photon & $\rightarrow$ & $\mathrm{H}+\mathrm{H}_{2}{ }^{+}$ \\
\hline $\mathrm{C}^{-}+$Photon & $\rightarrow$ & $\mathrm{C}+\mathrm{e}^{-}$ & $\mathrm{H}^{-}+$Photon & $\rightarrow$ & $\mathrm{H}+\mathrm{e}^{-}$ & $\mathrm{O}^{-}+$Photon & $\rightarrow$ & $\mathrm{O}+\mathrm{e}^{-}$ \\
\hline C + Photon & $\rightarrow$ & $\mathrm{C}^{+}+\mathrm{e}^{-}$ & $\mathrm{CO}+$ Photon & $\rightarrow$ & $\mathrm{C}+\mathrm{O}$ & $\mathrm{H}_{2}+$ Photon & $\rightarrow$ & $\mathrm{H}+\mathrm{H}$ \\
\hline $\mathrm{O}_{2}+$ Photon & $\rightarrow$ & $\mathrm{O}+\mathrm{O}$ & $\mathrm{OH}+$ Photon & $\rightarrow$ & $\mathrm{H}+\mathrm{O}$ & $\mathrm{OH}+$ Photon & $\rightarrow$ & $\mathrm{OH}^{+}+\mathrm{e}^{-}$ \\
\hline $\mathrm{CO}_{2}+$ Photon & $\rightarrow$ & $\mathrm{O}+\mathrm{CO}$ & $\mathrm{H}_{2} \mathrm{O}+$ Photon & $\rightarrow$ & $\mathrm{H}+\mathrm{OH}$ & $\mathrm{H}_{2} \mathrm{O}+$ Photon & $\rightarrow$ & $\mathrm{H}_{2} \mathrm{O}^{+}+\mathrm{e}^{-}$ \\
\hline $\mathrm{HCO}+$ Photon & $\rightarrow$ & $\mathrm{H}+\mathrm{CO}$ & HCO + Photon & $\rightarrow$ & $\mathrm{HCO}^{+}+\mathrm{e}^{-}$ & $\mathrm{H}_{2} \mathrm{CO}+$ Photon & $\rightarrow$ & $\mathrm{H}+\mathrm{H}+\mathrm{CO}$ \\
\hline $\mathrm{H}_{2} \mathrm{CO}+$ Photon & $\rightarrow$ & $\mathrm{CO}+\mathrm{H}_{2}$ & $\mathrm{H}_{2} \mathrm{CO}+$ Photon & $\rightarrow$ & $\mathrm{H}+\mathrm{HCO}^{+}+\mathrm{e}^{-}$ & & & \\
\hline
\end{tabular}

Table A.3. Gas-phase reactions.

\begin{tabular}{|c|c|c|c|c|c|c|c|c|}
\hline Reactants & & Products & Reactants & & Products & Reactants & & Products \\
\hline $\mathrm{H}_{2} \mathrm{O}+\mathrm{C}^{+}$ & $\rightarrow$ & $\mathrm{H}+\mathrm{HCO}^{+}$ & $\mathrm{O}_{2}+\mathrm{C}^{-}$ & $\rightarrow$ & $\mathrm{CO}+\mathrm{O}^{-}$ & $\mathrm{CO}_{2}+\mathrm{C}^{-}$ & $\rightarrow$ & $\mathrm{CO}+\mathrm{CO}+\mathrm{e}^{-}$ \\
\hline $\mathrm{O}+\mathrm{HCO}$ & $\rightarrow$ & $\mathrm{H}+\mathrm{CO}_{2}$ & $\mathrm{O}+\mathrm{HCO}$ & $\rightarrow$ & $\mathrm{CO}+\mathrm{OH}$ & $\mathrm{CO}+\mathrm{OH}$ & $\rightarrow$ & $\mathrm{H}+\mathrm{CO}_{2}$ \\
\hline $\mathrm{OH}+\mathrm{OH}$ & $\rightarrow$ & $\mathrm{O}+\mathrm{H}_{2} \mathrm{O}$ & $\mathrm{OH}+\mathrm{OH}$ & $\rightarrow$ & $\mathrm{O}+\mathrm{H}_{2} \mathrm{O}$ & $\mathrm{OH}+\mathrm{HCO}$ & $\rightarrow$ & $\mathrm{CO}+\mathrm{H}_{2} \mathrm{O}$ \\
\hline $\mathrm{OH}+\mathrm{H}_{2} \mathrm{CO}$ & $\rightarrow$ & $\mathrm{H}_{2} \mathrm{O}+\mathrm{HCO}$ & $\mathrm{O}+\mathrm{H}_{2}^{+}$ & $\rightarrow$ & $\mathrm{H}+\mathrm{OH}^{+}$ & $\mathrm{CO}+\mathrm{H}_{2}^{+}$ & $\rightarrow$ & $\mathrm{H}+\mathrm{HCO}^{+}$ \\
\hline $\mathrm{H}_{2}+\mathrm{H}_{2}^{+}$ & $\rightarrow$ & $\mathrm{H}+\mathrm{H}_{3}^{+}$ & $\mathrm{OH}+\mathrm{H}_{2}^{+}$ & $\rightarrow$ & $\mathrm{H}+\mathrm{H}_{2} \mathrm{O}^{+}$ & $\mathrm{H}_{2} \mathrm{O}+\mathrm{H}_{2}^{+}$ & $\rightarrow$ & $\mathrm{H}+\mathrm{H}_{3} \mathrm{O}^{+}$ \\
\hline $\mathrm{HCO}+\mathrm{H}_{2}{ }^{+}$ & $\rightarrow$ & $\mathrm{CO}+\mathrm{H}_{3}^{+}$ & $\mathrm{H}_{2} \mathrm{CO}+\mathrm{H}_{2}^{+}$ & $\rightarrow$ & $\mathrm{H}+\mathrm{H}_{2}+\mathrm{HCO}^{+}$ & $\mathrm{H}_{3}^{+}+\mathrm{H}^{-}$ & $\rightarrow$ & $\mathrm{H}_{2}+\mathrm{H}_{2}$ \\
\hline $\mathrm{HCO}^{+}+\mathrm{H}^{-}$ & $\rightarrow$ & $\mathrm{CO}+\mathrm{H}_{2}$ & $\mathrm{H}_{3} \mathrm{O}^{+}+\mathrm{H}^{-}$ & $\rightarrow$ & $\mathrm{H}+\mathrm{H}_{2}+\mathrm{OH}$ & $\mathrm{H}_{3} \mathrm{O}^{+}+\mathrm{H}^{-}$ & $\rightarrow$ & $\mathrm{H}_{2}+\mathrm{H}_{2} \mathrm{O}$ \\
\hline $\mathrm{OH}+\mathrm{HCO}^{+}$ & $\rightarrow$ & $\mathrm{CO}+\mathrm{H}_{2} \mathrm{O}^{+}$ & $\mathrm{H}_{2} \mathrm{O}+\mathrm{HCO}^{+}$ & $\rightarrow$ & $\mathrm{CO}+\mathrm{H}_{3} \mathrm{O}^{+}$ & $\mathrm{HCO}+\mathrm{HCO}$ & $\rightarrow$ & $\mathrm{CO}+\mathrm{H}_{2} \mathrm{CO}$ \\
\hline $\mathrm{H}+\mathrm{HCO}$ & $\rightarrow$ & $\mathrm{CO}+\mathrm{H}_{2}$ & $\mathrm{H}+\mathrm{OH}$ & $\rightarrow$ & $\mathrm{O}+\mathrm{H}_{2}$ & $\mathrm{H}+\mathrm{H}_{2} \mathrm{O}$ & $\rightarrow$ & $\mathrm{H}_{2}+\mathrm{OH}$ \\
\hline $\mathrm{H}+\mathrm{H}_{2} \mathrm{CO}$ & $\rightarrow$ & $\mathrm{H}_{2}+\mathrm{HCO}$ & $\mathrm{H}+\mathrm{O}_{2}$ & $\rightarrow$ & $\mathrm{O}+\mathrm{OH}$ & $\mathrm{H}+\mathrm{CO}_{2}$ & $\rightarrow$ & $\mathrm{CO}+\mathrm{OH}$ \\
\hline $\mathrm{O}+\mathrm{H}_{2}$ & $\rightarrow$ & $\mathrm{H}+\mathrm{OH}$ & $\mathrm{H}_{2}+\mathrm{OH}$ & $\rightarrow$ & $\mathrm{H}+\mathrm{H}_{2} \mathrm{O}$ & $\mathrm{O}+\mathrm{OH}$ & $\rightarrow$ & $\mathrm{H}+\mathrm{O}_{2}$ \\
\hline $\mathrm{O}+\mathrm{OH}$ & $\rightarrow$ & $\mathrm{H}+\mathrm{O}_{2}$ & $\mathrm{O}+\mathrm{OH}$ & $\rightarrow$ & $\mathrm{H}+\mathrm{O}_{2}$ & $\mathrm{CO}+\mathrm{H}_{2} \mathrm{O}^{+}$ & $\rightarrow$ & $\mathrm{OH}+\mathrm{HCO}^{+}$ \\
\hline $\mathrm{H}_{2}+\mathrm{H}_{2} \mathrm{O}^{+}$ & $\rightarrow$ & $\mathrm{H}+\mathrm{H}_{3} \mathrm{O}^{+}$ & $\mathrm{OH}+\mathrm{H}_{2} \mathrm{O}^{+}$ & $\rightarrow$ & $\mathrm{O}+\mathrm{H}_{3} \mathrm{O}^{+}$ & $\mathrm{H}_{2} \mathrm{O}+\mathrm{H}_{2} \mathrm{O}^{+}$ & $\rightarrow$ & $\mathrm{OH}+\mathrm{H}_{3} \mathrm{O}^{+}$ \\
\hline $\mathrm{HCO}+\mathrm{H}_{2} \mathrm{O}^{+}$ & $\rightarrow$ & $\mathrm{CO}+\mathrm{H}_{3} \mathrm{O}^{+}$ & $\mathrm{O}+\mathrm{H}_{3}{ }^{+}$ & $\rightarrow$ & $\mathrm{H}_{2}+\mathrm{OH}^{+}$ & $\mathrm{O}+\mathrm{H}_{3}{ }^{+}$ & $\rightarrow$ & $\mathrm{H}+\mathrm{H}_{2} \mathrm{O}^{+}$ \\
\hline $\mathrm{CO}+\mathrm{H}_{3}^{+}$ & $\rightarrow$ & $\mathrm{H}_{2}+\mathrm{HCO}^{+}$ & $\mathrm{OH}+\mathrm{H}_{3}{ }^{+}$ & $\rightarrow$ & $\mathrm{H}_{2}+\mathrm{H}_{2} \mathrm{O}^{+}$ & $\mathrm{H}_{2} \mathrm{O}+\mathrm{H}_{3}{ }^{+}$ & $\rightarrow$ & $\mathrm{H}_{2}+\mathrm{H}_{3} \mathrm{O}^{+}$ \\
\hline $\mathrm{CO}_{2}+\mathrm{H}^{+}$ & $\rightarrow$ & $\mathrm{O}+\mathrm{HCO}^{+}$ & $\mathrm{HCO}+\mathrm{H}^{+}$ & $\rightarrow$ & $\mathrm{CO}+\mathrm{H}_{2}^{+}$ & $\mathrm{H}_{2}+\mathrm{O}^{+}$ & $\rightarrow$ & $\mathrm{H}+\mathrm{OH}^{+}$ \\
\hline $\mathrm{HCO}+\mathrm{O}^{+}$ & $\rightarrow$ & $\mathrm{CO}+\mathrm{OH}^{+}$ & $\mathrm{H}_{2} \mathrm{CO}+\mathrm{O}^{+}$ & $\rightarrow$ & $\mathrm{OH}+\mathrm{HCO}^{+}$ & $\mathrm{C}+\mathrm{H}_{3} \mathrm{O}^{+}$ & $\rightarrow$ & $\mathrm{H}_{2}+\mathrm{HCO}^{+}$ \\
\hline $\mathrm{H}_{2} \mathrm{CO}+\mathrm{H}^{+}$ & $\rightarrow$ & $\mathrm{H}_{2}+\mathrm{HCO}^{+}$ & $\mathrm{CO}+\mathrm{OH}^{+}$ & $\rightarrow$ & $\mathrm{O}+\mathrm{HCO}^{+}$ & $\mathrm{H}_{2}+\mathrm{OH}^{+}$ & $\rightarrow$ & $\mathrm{H}+\mathrm{H}_{2} \mathrm{O}^{+}$ \\
\hline $\mathrm{OH}+\mathrm{OH}^{+}$ & $\rightarrow$ & $\mathrm{O}+\mathrm{H}_{2} \mathrm{O}^{+}$ & $\mathrm{H}_{2} \mathrm{O}+\mathrm{OH}^{+}$ & $\rightarrow$ & $\mathrm{O}+\mathrm{H}_{3} \mathrm{O}^{+}$ & $\mathrm{HCO}+\mathrm{OH}^{+}$ & $\rightarrow$ & $\mathrm{CO}+\mathrm{H}_{2} \mathrm{O}^{+}$ \\
\hline $\mathrm{C}+\mathrm{O}_{2}$ & $\rightarrow$ & $\mathrm{O}+\mathrm{CO}$ & $\mathrm{C}+\mathrm{OH}$ & $\rightarrow$ & $\mathrm{H}+\mathrm{CO}$ & $\mathrm{HCO}^{+}+\mathrm{C}^{-}$ & $\rightarrow$ & $\mathrm{C}+\mathrm{H}+\mathrm{CO}$ \\
\hline $\mathrm{H}_{3} \mathrm{O}^{+}+\mathrm{C}^{-}$ & $\rightarrow$ & $\mathrm{C}+\mathrm{H}+\mathrm{H}_{2} \mathrm{O}$ & $\mathrm{H}_{3}{ }^{+}+\mathrm{C}^{-}$ & $\rightarrow$ & $\mathrm{C}+\mathrm{H}+\mathrm{H}_{2}$ & $\mathrm{HCO}^{+}+\mathrm{O}^{-}$ & $\rightarrow$ & $\mathrm{H}+\mathrm{O}+\mathrm{CO}$ \\
\hline $\mathrm{H}_{3} \mathrm{O}^{+}+\mathrm{O}^{-}$ & $\rightarrow$ & $\mathrm{H}+\mathrm{O}+\mathrm{H}_{2} \mathrm{O}$ & $\mathrm{H}_{3}^{+}+\mathrm{O}^{-}$ & $\rightarrow$ & $\mathrm{H}+\mathrm{O}+\mathrm{H}_{2}$ & $\mathrm{H}+\mathrm{H}_{2}{ }^{+}$ & $\rightarrow$ & $\mathrm{H}_{2}+\mathrm{H}^{+}$ \\
\hline $\mathrm{C}^{+}+\mathrm{C}^{-}$ & $\rightarrow$ & $\mathrm{C}+\mathrm{C}$ & $\mathrm{C}^{+}+\mathrm{H}^{-}$ & $\rightarrow$ & $\mathrm{C}+\mathrm{H}$ & $\mathrm{H}^{+}+\mathrm{C}^{-}$ & $\rightarrow$ & $\mathrm{C}+\mathrm{H}$ \\
\hline $\mathrm{H}^{+}+\mathrm{H}^{-}$ & $\rightarrow$ & $\mathrm{H}+\mathrm{H}$ & $\mathrm{O}^{+}+\mathrm{C}^{-}$ & $\rightarrow$ & $\mathrm{C}+\mathrm{O}$ & $\mathrm{O}^{+}+\mathrm{H}^{-}$ & $\rightarrow$ & $\mathrm{H}+\mathrm{O}$ \\
\hline $\mathrm{H}_{2}{ }^{+}+\mathrm{H}^{-}$ & $\rightarrow$ & $\mathrm{H}+\mathrm{H}_{2}$ & $\mathrm{O}+\mathrm{H}^{+}$ & $\rightarrow$ & $\mathrm{H}+\mathrm{O}^{+}$ & $\mathrm{OH}+\mathrm{H}^{+}$ & $\rightarrow$ & $\mathrm{H}+\mathrm{OH}^{+}$ \\
\hline $\mathrm{H}_{2} \mathrm{O}+\mathrm{H}^{+}$ & $\rightarrow$ & $\mathrm{H}+\mathrm{H}_{2} \mathrm{O}^{+}$ & $\mathrm{H}+\mathrm{O}^{+}$ & $\rightarrow$ & $\mathrm{O}+\mathrm{H}^{+}$ & $\mathrm{H}_{2} \mathrm{O}+\mathrm{O}^{+}$ & $\rightarrow$ & $\mathrm{O}+\mathrm{H}_{2} \mathrm{O}^{+}$ \\
\hline $\mathrm{HCO}+\mathrm{C}^{+}$ & $\rightarrow$ & $\mathrm{C}+\mathrm{HCO}^{+}$ & $\mathrm{HCO}+\mathrm{O}^{+}$ & $\rightarrow$ & $\mathrm{O}+\mathrm{HCO}^{+}$ & $\mathrm{OH}+\mathrm{O}^{+}$ & $\rightarrow$ & $\mathrm{O}+\mathrm{OH}^{+}$ \\
\hline $\mathrm{OH}+\mathrm{H}_{2}^{+}$ & $\rightarrow$ & $\mathrm{H}_{2}+\mathrm{OH}^{+}$ & $\mathrm{H}_{2} \mathrm{O}+\mathrm{H}_{2}{ }^{+}$ & $\rightarrow$ & $\mathrm{H}_{2}+\mathrm{H}_{2} \mathrm{O}^{+}$ & $\mathrm{HCO}+\mathrm{H}_{2}{ }^{+}$ & $\rightarrow$ & $\mathrm{H}_{2}+\mathrm{HCO}^{+}$ \\
\hline $\mathrm{HCO}+\mathrm{H}_{2} \mathrm{O}^{+}$ & $\rightarrow$ & $\mathrm{H}_{2} \mathrm{O}+\mathrm{HCO}^{+}$ & $\mathrm{HCO}+\mathrm{H}^{+}$ & $\rightarrow$ & $\mathrm{H}+\mathrm{HCO}^{+}$ & $\mathrm{H}_{2} \mathrm{O}+\mathrm{OH}^{+}$ & $\rightarrow$ & $\mathrm{OH}+\mathrm{H}_{2} \mathrm{O}^{+}$ \\
\hline $\mathrm{HCO}+\mathrm{OH}^{+}$ & $\rightarrow$ & $\mathrm{OH}+\mathrm{HCO}^{+}$ & $\mathrm{HCO}^{+}+\mathrm{C}^{-}$ & $\rightarrow$ & $\mathrm{C}+\mathrm{HCO}$ & $\mathrm{HCO}^{+}+\mathrm{O}^{-}$ & $\rightarrow$ & $\mathrm{O}+\mathrm{HCO}$ \\
\hline $\mathrm{H}^{+}+\mathrm{O}^{-}$ & $\rightarrow$ & $\mathrm{H}+\mathrm{O}$ & $\mathrm{H}+\mathrm{H}^{+}$ & $\rightarrow$ & $\mathrm{H}_{2}{ }^{+}+$Photon & $\mathrm{C}+\mathrm{O}$ & $\rightarrow$ & $\mathrm{CO}+$ Photon \\
\hline $\mathrm{H}+\mathrm{O}$ & $\rightarrow$ & $\mathrm{OH}+$ Photon & $\mathrm{O}+\mathrm{O}$ & $\rightarrow$ & $\mathrm{O}_{2}+$ Photon & $\mathrm{H}+\mathrm{OH}$ & $\rightarrow$ & $\mathrm{H}_{2} \mathrm{O}+$ Photon \\
\hline $\mathrm{O}+\mathrm{C}^{-}$ & $\rightarrow$ & $\mathrm{CO}+\mathrm{e}^{-}$ & $\mathrm{O}_{2}+\mathrm{C}^{-}$ & $\rightarrow$ & $\mathrm{CO}_{2}+\mathrm{e}^{-}$ & $\mathrm{OH}+\mathrm{C}^{-}$ & $\rightarrow$ & $\mathrm{HCO}+\mathrm{e}^{-}$ \\
\hline $\mathrm{H}_{2} \mathrm{O}+\mathrm{C}^{-}$ & $\rightarrow$ & $\mathrm{H}_{2} \mathrm{CO}+\mathrm{e}^{-}$ & $\mathrm{H}+\mathrm{H}^{-}$ & $\rightarrow$ & $\mathrm{H}_{2}+\mathrm{e}^{-}$ & $\mathrm{O}+\mathrm{H}^{-}$ & $\rightarrow$ & $\mathrm{OH}+\mathrm{e}^{-}$ \\
\hline $\mathrm{CO}+\mathrm{H}^{-}$ & $\rightarrow$ & $\mathrm{HCO}+\mathrm{e}^{-}$ & $\mathrm{OH}+\mathrm{H}^{-}$ & $\rightarrow$ & $\mathrm{H}_{2} \mathrm{O}+\mathrm{e}^{-}$ & $\mathrm{HCO}+\mathrm{H}^{-}$ & $\rightarrow$ & $\mathrm{H}_{2} \mathrm{CO}+\mathrm{e}^{-}$ \\
\hline $\mathrm{C}+\mathrm{O}^{-}$ & $\rightarrow$ & $\mathrm{CO}+\mathrm{e}^{-}$ & $\mathrm{H}+\mathrm{O}^{-}$ & $\rightarrow$ & $\mathrm{OH}+\mathrm{e}^{-}$ & $\mathrm{O}+\mathrm{O}^{-}$ & $\rightarrow$ & $\mathrm{O}_{2}+\mathrm{e}^{-}$ \\
\hline $\mathrm{CO}+\mathrm{O}^{-}$ & $\rightarrow$ & $\mathrm{CO}_{2}+\mathrm{e}^{-}$ & $\mathrm{H}_{2}+\mathrm{O}^{-}$ & $\rightarrow$ & $\mathrm{H}_{2} \mathrm{O}+\mathrm{e}^{-}$ & $\mathrm{C}+\mathrm{e}^{-}$ & $\rightarrow$ & $\mathrm{C}^{-}$ \\
\hline $\mathrm{H}+\mathrm{e}^{-}$ & $\rightarrow$ & $\mathrm{H}^{-}$ & $\mathrm{O}+\mathrm{e}^{-}$ & $\rightarrow$ & $\mathrm{O}^{-}$ & $\mathrm{H}_{2}^{+}+\mathrm{e}^{-}$ & $\rightarrow$ & $\mathrm{H}+\mathrm{H}$ \\
\hline $\mathrm{H}_{2}^{+}+\mathrm{e}^{-}$ & $\rightarrow$ & $\mathrm{H}_{2}+$ Photon & $\mathrm{OH}^{+}+\mathrm{e}^{-}$ & $\rightarrow$ & $\mathrm{H}+\mathrm{O}$ & $\mathrm{H}_{2} \mathrm{O}^{+}+\mathrm{e}^{-}$ & $\rightarrow$ & $\mathrm{O}+\mathrm{H}_{2}$ \\
\hline $\mathrm{H}_{2} \mathrm{O}^{+}+\mathrm{e}^{-}$ & $\rightarrow$ & $\mathrm{H}+\mathrm{OH}$ & $\mathrm{H}_{2} \mathrm{O}^{+}+\mathrm{e}^{-}$ & $\rightarrow$ & $\mathrm{H}+\mathrm{H}+\mathrm{O}$ & $\mathrm{H}_{3}^{+}+\mathrm{e}^{-}$ & $\rightarrow$ & $\mathrm{H}+\mathrm{H}+\mathrm{H}$ \\
\hline $\mathrm{H}_{3}^{+}+\mathrm{e}^{-}$ & $\rightarrow$ & $\mathrm{H}+\mathrm{H}_{2}$ & $\mathrm{HCO}^{+}+\mathrm{e}^{-}$ & $\rightarrow$ & $\mathrm{H}+\mathrm{CO}$ & $\mathrm{C}^{+}+\mathrm{e}^{-}$ & $\rightarrow$ & C + Photon \\
\hline $\mathrm{H}^{+}+\mathrm{e}^{-}$ & $\rightarrow$ & $\mathrm{H}+$ Photon & $\mathrm{O}^{+}+\mathrm{e}^{-}$ & $\rightarrow$ & $\mathrm{O}+$ Photon & $\mathrm{H}_{3} \mathrm{O}^{+}+\mathrm{e}^{-}$ & $\rightarrow$ & $\mathrm{H}+\mathrm{H}+\mathrm{OH}$ \\
\hline $\mathrm{H}_{3} \mathrm{O}^{+}+\mathrm{e}^{-}$ & $\rightarrow$ & $\mathrm{H}+\mathrm{H}_{2} \mathrm{O}$ & $\mathrm{H}_{3} \mathrm{O}^{+}+\mathrm{e}^{-}$ & $\rightarrow$ & $\mathrm{H}_{2}+\mathrm{OH}$ & $\mathrm{H}_{3} \mathrm{O}^{+}+\mathrm{e}^{-}$ & $\rightarrow$ & $\mathrm{H}+\mathrm{O}+\mathrm{H}_{2}$ \\
\hline $\mathrm{O}_{2}+\mathrm{C}^{+}$ & $\rightarrow$ & $\mathrm{CO}+\mathrm{O}^{+}$ & & & & & & \\
\hline
\end{tabular}


S. Hocuk and S. Cazaux: Interplay of gas and ice during cloud evolution

Table A.4. Surface reactions leading to surface products.

\begin{tabular}{|c|c|c|c|c|}
\hline Reactants & & Products & $E_{\mathrm{a}} / \mathrm{K}$ & Reference \\
\hline$\perp \mathrm{H}+\perp \mathrm{H}$ & $\rightarrow$ & $\perp \mathrm{H}_{2}$ & 0 & \\
\hline$\perp \mathrm{H}+\perp \mathrm{O}$ & $\rightarrow$ & $\perp \mathrm{OH}$ & 0 & \\
\hline$\perp \mathrm{H}+\perp \mathrm{OH}$ & $\rightarrow$ & $\perp \mathrm{H}_{2} \mathrm{O}$ & 0 & \\
\hline$\perp \mathrm{H}+\perp \mathrm{O}_{3}$ & $\rightarrow$ & $\perp \mathrm{OH}+\perp \mathrm{O}_{2}$ & 480 & c \\
\hline$\perp \mathrm{H}+\perp \mathrm{O}_{3}$ & $\rightarrow$ & $\perp \mathrm{OH}+\perp \mathrm{O}_{2}$ & & $\mathrm{i}, \mathrm{a}(450)$ \\
\hline$\perp \mathrm{H}+\perp \mathrm{H}_{2} \mathrm{O}_{2}$ & $\rightarrow$ & $\perp \mathrm{OH}+\perp \mathrm{H}_{2} \mathrm{O}$ & 1000 & $\mathrm{o}(800-1250)$ \\
\hline$\perp \mathrm{H}+\perp \mathrm{O}_{2}$ & $\rightarrow$ & $\perp \mathrm{HO}_{2}$ & 200 & $\mathrm{~b}(0-250)$ \\
\hline$\perp \mathrm{H}+\perp \mathrm{O}_{2}$ & $\rightarrow$ & $\perp \mathrm{HO}_{2}$ & & $\mathrm{j}(0-200)$ \\
\hline$\perp \mathrm{H}+\perp \mathrm{H}_{2} \mathrm{O}$ & $\rightarrow$ & $\perp \mathrm{OH}+\perp \mathrm{H}_{2}$ & 9600 & $r$ \\
\hline$\perp \mathrm{H}+\perp \mathrm{HO}_{2}$ & $\rightarrow$ & $\perp \mathrm{OH}+\perp \mathrm{OH}$ & 0 & \\
\hline$\perp \mathrm{H}+\perp \mathrm{CO}$ & $\rightarrow$ & $\perp \mathrm{HCO}$ & 600 & $\mathrm{~h}, \mathrm{k}$ \\
\hline$\perp \mathrm{H}+\perp \mathrm{HCO}$ & $\rightarrow$ & $\perp \mathrm{H}_{2} \mathrm{CO}$ & 0 & \\
\hline$\perp \mathrm{H}+\perp \mathrm{H}_{2} \mathrm{CO}$ & $\rightarrow$ & $\perp \mathrm{CH}_{3} \mathrm{O}$ & 400 & $\mathrm{~h}, \mathrm{k}$ \\
\hline$\perp \mathrm{H}+\perp \mathrm{CH}_{3} \mathrm{O}$ & $\rightarrow$ & $\perp \mathrm{CH}_{3} \mathrm{OH}$ & 0 & \\
\hline$\perp \mathrm{H}+\perp \mathrm{HCO}$ & $\rightarrow$ & $\perp \mathrm{CO}+\perp \mathrm{H}_{2}$ & 400 & $\mathrm{q}$ \\
\hline$\perp \mathrm{H}+\perp \mathrm{H}_{2} \mathrm{CO}$ & $\rightarrow$ & $\perp \mathrm{HCO} \perp \mathrm{H}_{2}$ & 2250 & $\mathrm{n}$ \\
\hline$\perp \mathrm{H}+\perp \mathrm{CH}_{3} \mathrm{O}$ & $\rightarrow$ & $\perp \mathrm{H}_{2} \mathrm{CO}+\perp \mathrm{H}_{2}$ & 150 & $\mathrm{n}$ \\
\hline$\perp \mathrm{H}+\perp \mathrm{CH}_{3} \mathrm{OH}$ & $\rightarrow$ & $\perp \mathrm{CH}_{3} \mathrm{O}+\perp \mathrm{H}_{2}$ & 3000 & $\mathrm{n}$ \\
\hline$\perp \mathrm{H}+\perp \mathrm{CO}_{2}$ & $\rightarrow$ & $\perp \mathrm{CO}+\perp \mathrm{OH}$ & 10000 & $\mathrm{n}$ \\
\hline$\perp \mathrm{O}+\perp \mathrm{O}$ & $\rightarrow$ & $\perp \mathrm{O}_{2}$ & 0 & \\
\hline$\perp \mathrm{O}+\perp \mathrm{O}_{2}$ & $\rightarrow$ & $\perp \mathrm{O}_{3}$ & 0 & \\
\hline$\perp \mathrm{O}+\perp \mathrm{O}_{3}$ & $\rightarrow$ & $\perp \mathrm{O}_{2}+\perp \mathrm{O}_{2}$ & 2300 & $\mathrm{p}$ \\
\hline$\perp \mathrm{O}+\perp \mathrm{O}_{3}$ & $\rightarrow$ & $\perp \mathrm{O}_{2}+\perp \mathrm{O}_{2}$ & & $\mathrm{r}(2000)$ \\
\hline$\perp \mathrm{O}+\perp \mathrm{HO}_{2}$ & $\rightarrow$ & $\perp \mathrm{O}_{2}+\perp \mathrm{OH}$ & 0 & \\
\hline$\perp \mathrm{O}+\perp \mathrm{H}_{2}$ & $\rightarrow$ & $\perp \mathrm{H}+\perp \mathrm{OH}$ & 4640 & 1 \\
\hline$\perp \mathrm{O}+\perp \mathrm{OH}$ & $\rightarrow$ & $\perp \mathrm{O}_{2}+\perp \mathrm{H}$ & 0 & \\
\hline$\perp \mathrm{O}+\perp \mathrm{CO}$ & $\rightarrow$ & $\perp \mathrm{CO}_{2}$ & 160 & $\mathrm{p}(\geq 160)$ \\
\hline$\perp \mathrm{O}+\perp \mathrm{CO}$ & $\rightarrow$ & $\perp \mathrm{CO}_{2}$ & & $\mathrm{e}(290)$ \\
\hline$\perp \mathrm{O}+\perp \mathrm{HCO}$ & $\rightarrow$ & $\perp \mathrm{CO}_{2}+\perp \mathrm{H}$ & 0 & \\
\hline$\perp \mathrm{O}+\perp \mathrm{H}_{2} \mathrm{CO}$ & $\rightarrow$ & $\perp \mathrm{CO}_{2}+\perp \mathrm{H}_{2}$ & 300 & $\mathrm{q}$ \\
\hline$\perp \mathrm{OH}+\perp \mathrm{OH}$ & $\rightarrow$ & $\perp \mathrm{H}_{2} \mathrm{O}_{2}$ & 0 & \\
\hline$\perp \mathrm{OH}+\perp \mathrm{H}_{2}$ & $\rightarrow$ & $\perp \mathrm{H}+\perp \mathrm{H}_{2} \mathrm{O}$ & 2100 & $\mathrm{~g}$ \\
\hline$\perp \mathrm{OH}+\perp \mathrm{CO}$ & $\rightarrow$ & $\perp \mathrm{CO}_{2}+\perp \mathrm{H}$ & 600 & $\mathrm{~d}$ \\
\hline$\perp \mathrm{OH}+\perp \mathrm{CO}$ & $\rightarrow$ & $\perp \mathrm{CO}_{2}+\perp \mathrm{H}$ & & $\mathrm{f}(519), \mathrm{m}(400)$ \\
\hline$\perp \mathrm{OH}+\perp \mathrm{HCO}$ & $\rightarrow$ & $\perp \mathrm{CO}_{2}+\perp \mathrm{H}_{2}$ & 0 & \\
\hline$\perp \mathrm{HO}_{2}+\perp \mathrm{H}_{2}$ & $\rightarrow$ & $\perp \mathrm{H}+\perp \mathrm{H}_{2} \mathrm{O}_{2}$ & 5000 & $\mathrm{o}$ \\
\hline
\end{tabular}

Notes. Note 1: The parentheses give barriers from respective studies. Note 2: Extra references given for reactions with similar barriers. Note 3: For unknown/uncertain barriers, the NIST ${ }^{n}$ database is used. Note 4: The barriers for $\perp \mathrm{H}+\perp \mathrm{CO}$ and $\perp \mathrm{H}+\perp \mathrm{H}_{2} \mathrm{CO}$ are "effective" barriers deduced from $h, k$ using their rates.

References. a: Lee et al. (1978), b: Walch et al. (1988); c: Atkinson et al. (1989); d: Duncan \& Miller (2000); e: Roser et al. (2001); f: Yu et al. (2001); g: Atkinson et al. (2004); h: Awad et al. (2005); i: Cuppen \& Herbst (2007); j: Miyauchi et al. (2008); k: Fuchs et al. (2009); 1: Agúndez et al. (2010); m: Noble et al. (2011); n: NIST database; http://kinetics.nist.gov; o: Lamberts et al. (2013); p: Minissale et al. (2013b); q: Best estimate (priv. comm. Minissale); r: Cazaux et al. (2010).
Table A.5. Chemical desorption reactions.

\begin{tabular}{lll|c|l}
\hline \hline Reactants & & Products & $E_{\mathrm{a}} / \mathrm{K}$ & Reference \\
\hline$\perp \mathrm{H}+\perp \mathrm{H}$ & $\rightarrow$ & $\mathrm{H}_{2}$ & 0 & \\
$\perp \mathrm{H}+\perp \mathrm{O}$ & $\rightarrow$ & $\mathrm{OH}$ & 0 & \\
$\perp \mathrm{H}+\perp \mathrm{OH}$ & $\rightarrow$ & $\mathrm{H}_{2} \mathrm{O}$ & 0 & \\
$\perp \mathrm{H}+\perp \mathrm{O}_{3}$ & $\rightarrow$ & $\mathrm{OH}+\mathrm{O}_{2}$ & 480 & See Table A.4 \\
$\perp \mathrm{H}+\perp \mathrm{H}_{2} \mathrm{O}_{2}$ & $\rightarrow$ & $\mathrm{OH}+\mathrm{H}_{2} \mathrm{O}$ & 1000 & See Table A.4 \\
$\perp \mathrm{H}+\perp \mathrm{CO}$ & $\rightarrow$ & $\mathrm{HCO}$ & 600 & See Table A.4 \\
$\perp \mathrm{H}+\perp \mathrm{HCO}$ & $\rightarrow$ & $\mathrm{H}_{2} \mathrm{CO}$ & 0 & \\
$\perp \mathrm{H}+\perp \mathrm{H}_{2} \mathrm{CO}$ & $\rightarrow$ & $\mathrm{CH}_{3} \mathrm{O}$ & 400 & See Table A.4 \\
$\perp \mathrm{H}+\perp \mathrm{CH}_{3} \mathrm{O}$ & $\rightarrow$ & $\mathrm{CH}_{3} \mathrm{OH}$ & 0 & \\
$\perp \mathrm{O}+\perp \mathrm{O}$ & $\rightarrow$ & $\mathrm{O}_{2}$ & 0 & \\
$\perp \mathrm{O}+\perp \mathrm{O}_{3}$ & $\rightarrow$ & $\mathrm{O}_{2}+\mathrm{O}_{2}$ & 0 & \\
$\perp \mathrm{O}+\perp \mathrm{HO}_{2}$ & $\rightarrow$ & $\mathrm{O}_{2}+\mathrm{OH}$ & 0 & \\
\hline
\end{tabular}

Table A.6. Chemisorption reactions.

\begin{tabular}{ccc|c}
\hline \hline Reactants & & Products & $E_{\mathrm{a}} / \mathrm{K}$ \\
\hline $\mathrm{H}$ & $\rightarrow$ & $\perp \mathrm{H}_{c}$ & 1000 \\
$\mathrm{H}+\perp \mathrm{H}_{c}$ & $\rightarrow$ & $\mathrm{H}_{2}$ & 1000 \\
$\perp \mathrm{H}$ & $\rightarrow$ & $\perp \mathrm{H}_{c}$ & 10000 \\
$\perp \mathrm{H}_{c}$ & $\rightarrow$ & $\mathrm{H}$ & see Cazaux \& Tielens (2010)
\end{tabular}

$\perp \mathrm{H}_{c} \quad \rightarrow \quad \mathrm{H} \quad$ see Cazaux \& Tielens (2010)

$\perp \mathrm{H}+\perp \mathrm{H}_{c} \quad \rightarrow \quad \mathrm{H}_{2} \quad$ see Cazaux \& Tielens (2010) 